Article

\title{
The Heterogeneous Relationship between Pollution Charges and Enterprise Green Technology Innovation, Based on the Data of Chinese Industrial Enterprises
}

\author{
Mingyue Wang ${ }^{1}$, Yingming $\mathrm{Li}^{1,2, *}$, Zitong Wang ${ }^{1,2}$ and Junqiang $\mathrm{Li}^{3, *}$ \\ 1 Institutes of Science and Development, Chinese Academy of Sciences, No. 15, Zhongguancun Beiyitiao, \\ Haidian District, Beijing 100190, China; wangmingyue@casisd.cn (M.W.); zitongwangucas@126.com (Z.W.) \\ 2 School of Public Policy and Management, Chinese Academy of Sciences, No. 19(A) Yuquan Road, \\ Shijingshan District, Beijing 100049, China \\ 3 School of Economics and Management, Tongji University, Tongji Building A, Siping Road 1500, \\ Yangpu District, Shanghai 200092, China \\ * Correspondence: liyingming@casisd.cn (Y.L.); ljq@tongji.edu.cn (J.L.)
}

check for

updates

Citation: Wang, M.; Li, Y.; Wang, Z.;

Li, J. The Heterogeneous Relationship between Pollution Charges and Enterprise Green Technology Innovation, Based on the Data of Chinese Industrial Enterprises. Energies 2022, 15, 1663. https:// doi.org/10.3390/en15051663

Academic Editors: Grzegorz Mentel and Sebastian Majewski

Received: 20 January 2022

Accepted: 17 February 2022

Published: 23 February 2022

Publisher's Note: MDPI stays neutral with regard to jurisdictional claims in published maps and institutional affiliations.

Copyright: () 2022 by the authors. Licensee MDPI, Basel, Switzerland. This article is an open access article distributed under the terms and conditions of the Creative Commons Attribution (CC BY) license (https:/ / creativecommons.org/licenses/by/ $4.0 /)$.

\begin{abstract}
Enterprises' green technology innovation is critical to achieving the "win-win" of enterprise competitiveness and environmental protection. The impact of environmental regulation on green technology innovation by enterprises has been widely considered, but the conclusion has not yet been determined, and needs to be studied in detail. To this end, we studied the impact of pollution charge policy on different types of green technology innovation by industrial enterprises in China. We found that (1) the impact of pollution charges on most types of green technology innovation by enterprises has increased significantly over time; (2) the pollution charge policy has a certain inhibition effect on the end-of-pipe technology innovation, but can promote the process improvement of reducing industrial wastewater emissions; (3) there is a $U$-shaped relationship between the pollution charges and some green technological innovation (e.g., emission intensity of $\mathrm{SO}_{2}$, industrial wastewater emission intensity, and industrial wastewater removal intensity), which is dynamically adjusted over time; and (4) the larger the enterprise's solid assets, the faster the asset depreciation will inhibit the enterprise from adopting the green process innovation strategy.
\end{abstract}

Keywords: pollution charges; green technology innovation; industrial enterprise; heterogeneity analysis

\section{Introduction}

In 2019, China's GDP reached CNY 99,086.5 billion. With the rapid economic development, this surge was accompanied by a similarly prodigious growth in energy consumption, air pollution, and air-pollution-related deaths [1,2]. According to the Bulletin of China's Ecological Environment, in 2018, 121 of the 338 cities in China achieved ambient air quality standards, accounting for only $35.8 \%$. Resolving the uncoordinated relationship between economy and environment is an important factor in high-quality development and the construction of ecological civilization [3-5]. We can no longer rely on investment-driven innovation, and must turn to environmentally friendly technological innovation [6-8]. Enterprises are not only the core drivers of social and economic wealth creation, but also the most critical factors in the coordination of economic development and environmental protection $[9,10]$. Unfortunately, most of the existing studies on the economic effectiveness of environmental regulation are from the perspective of government and society, while there is little literature investigating micro-enterprises and macro-policy from the system perspective [11,12]. Therefore, in-depth exploration of how enterprises deal with environmental regulation is of great significance for the implementation of environmental regulation policies and the improvement of the green competitiveness of enterprises. 
The key to encouraging enterprises to participate in environmental management is to internalize externalities, including charging enterprises for polluting the environment and subsidizing enterprises for reducing pollution $[13,14]$. Pollution charges are an important policy tool of China's current environmental regulation system $[15,16]$. The policy basis is the Administrative Regulations on Pollution Discharge Fee Levy (http: / / www.gov.cn/zhengce/content/2008-03/28/content_5152.htm) (accessed on 12 May 2021), the Environmental Protection Law of the People's Republic of China (http:/ / mee.gov. cn/ywgz/fgbz/fl/201811/t20181114_673632.shtml) (accessed on 12 May 2021), and the Several Provisions on Strengthening the Management of Environmental Protection Subsidies (http:/ / www.csrcare.com/Law/Show?id=11782) (accessed on 12 May 2021). The purpose of pollution charges and environmental protection subsidies is to bring environmental factors into the production decision-making function of enterprise managers, so as to realize the internalization of the externality of environmental pollution $[17,18]$. While these environmental governance activities increase the additional costs faced by the enterprise and reduce the financial return of the shareholders [19], more and more studies have come to the conclusion that green technology innovation helps enterprises to reduce environmental costs, gain the trust of suppliers and customers, and seize the green competitive advantage [20-22]. Unlike direct participation in environmental governance and environmental protection investment, green technology innovation can not only reduce environmental pollution and improve environmental performance, but more importantly, green technology innovation enables enterprises to produce green-differentiated products, stimulate new market demand, and effectively improve their green competitiveness, so as to truly realize the "win-win" of economic efficiency and environmental protection [23,24].

Based on the above analysis, it is reasonable to believe that pollution charges have a certain influence on enterprises' green technology innovation, which is affected by factors such as charge intensity, types of green technology innovation, etc. The purpose of this article is to further reveal the multiple heterogeneity between pollution charges and green technology innovation from the level of Chinese industrial enterprises, including testing the impact of pollution charges on different types of green technology innovation, whether or not the relationship between them is, and the manifestations of the above relationship in different years. The rest of the paper is organized as follows: In Section 2, we review the literature on the relationship between pollution charges and green technology innovation. In Section 3, we describe our methodology in detail. Section 4 presents and discusses the empirical results. Section 5 concludes the paper and provides management implications.

\section{Literature Review}

The Nobel laureate in Economics Paul Romer pointed out that input factors and technological progress are the two main factors restricting economic growth [25]. Green technology innovation can reduce environmental pollution, save energy, and realize green, sustainable development, which coordinated between environmental protection and enterprise competitiveness [26-29]. No matter the general technological innovation or green technological innovation, innovation activities have the characteristics of long cycle, high investment, and high risk [30,31]. Therefore, the intensity of enterprises engaging in innovation activities depends on the managers' judgment of the risks and expected benefits of enterprise innovation activities [32]. However, the technological uncertainty, market uncertainty, and imperfect management system lead to the lack of motivation and ability in terms of enterprise innovation $[10,33]$. In order to minimize the negative impact of enterprise production on the environment, resources, and society, the government has adopted a series of environmental regulation measures, including administrative means [11,34], legal measures [35,36], and economic measures [37,38] (Cohen et al., 2017; Wang et al. 2020).

Pollution charges are a system wherein the state collects charges from organizations or individuals that discharge pollutants [39]. The neoclassical school believes that pollution charges increase the cost of enterprise compliance, as enterprises need to pay fees for their behavior of polluting the environment in the production process, increasing the capital bur- 
den on enterprises and occupying the resources of their green technology innovation [40]. Green technology innovation depends on a large amount of resource investment, and it takes a long time to show its positive impact on enterprise energy conservation, emissions reduction, and performance improvement [41]. Under the pressure of short-term performance and cash flow caused by pollution charges, managers are forced to give up green technology innovation with high investment, high risk, and high uncertainty [42].

Pollution charges as a regulation can force enterprises to adopt the strategy of green technology innovation, and provide compensatory benefits exceeding the cost of environmental regulation [43]. Enterprises can reduce their dependence on the original polluting production mode and effectively avoid the cost of pollution charges by applying green technology innovation to their production process [44]. The forced effect of pollution charges on enterprises' green technology innovation is reflected in the external pressure of stakeholders and the incentive factors within the enterprise.

In terms of external pressure, green development is the realistic appeal of external stakeholders to heavily polluting enterprises [45]. The Administrative Regulations on Pollution Discharge Fee Levy implemented in 2003 and the Environmental Protection Law of the People's Republic of China implemented in 2018 clearly stipulate that pollution charges shall be levied on the pollutant emissions of enterprises according to the pollution equivalent. Henriques and Sadorsky [46] found that stakeholders' pressure forces managers to weigh the consequences of environmental pollution, affecting the ways managers respond to pollution charges. Therefore, green technology innovation can enhance stakeholders' confidence in enterprises' green development, reduce stakeholders' negative expectations of enterprises' environmental pollution, and encourage managers to adopt a green innovation strategy in response to the demands of external stakeholders [47].

Although pollution charges constitute a cost for enterprises, and directly reduce their attainable profits, they can also encourage managers to actively reflect on the shortcomings of enterprises' own green development [48], effectively making up for the inherent defects of enterprises' governance mechanisms and overcoming the inertia of organizations not thinking about change [49]. Through green technology innovation, enterprises can not only realize the social benefits of energy conservation and emissions reduction, but also produce more green-differentiated products than their competitors, so as to obtain an increased market share and cultivate unique green competitive advantages [50,51]. In view of the above analysis, it can be assumed that pollution charges will encourage enterprises managers to carry out green technology innovation.

It can be seen from the above that the research on environmental regulation and green technology innovation covers a wide range, and it is very important to clarify the attributes of the research object, or else it may reach biased conclusions. At present, more and more quantitative studies are describing the relationship between environmental regulation and green technology innovation more accurately, by subdividing the types of environmental regulation, green technology innovation, and enterprise. Under the theoretical framework of the Porter hypothesis, the unique advantages of green technology innovation encourage enterprise managers to incorporate green and sustainable innovation schemes into their business decisions and strategic plans [52]. The pressure of pollution charges and the demands of stakeholders more actively encourage managers to carry out green technology innovation.

\section{Methodology}

\subsection{Data}

The basic data used in this research come from China's Industrial Enterprise Database and China's Industrial Enterprise Pollution Emission Database, both of which are maintained by the National Bureau of Statistics of China. The databases include all state-owned enterprises and non-state-owned enterprises above a designated size (with an annual main business income of more than CNY 5 million). In view of the availability and reliability of China's Industrial Enterprise Database and China's Industrial Enterprise Pollution Emis- 
sion Database, we mainly use the data from 2007 and 2012, because the latest data from China's Industrial Enterprise Database are for 2013, but the quality of the 2013 data is poor. China's Industrial Enterprise Database mainly includes basic information on enterprises and their main financial statistical indicators. China's Industrial Enterprise Pollution Emission Database mainly includes basic information on enterprises, consumption data for various energy resources, emission information on various pollutants, etc. Firstly, we matched China's Industrial Enterprise Database and China's Industrial Enterprise Pollution Emission Database of the corresponding year with the organization code and enterprise name, respectively. Then, the data obtained by matching the organization code and the data obtained by matching the enterprise name were integrated to delete duplicate samples, so as to obtain the original analysis data for the corresponding year. The original database with successful matching contains information on 47,172 enterprises in 2007 and 51,385 enterprises in 2012.

In view of the absence, omission, and error of some enterprises' observed values in the two databases used, before the data regression analysis, the sample data were processed as follows by referring to the practices of Brandt et al. [53] and Zhang et al. [54]: (1) exclude enterprise samples seriously lacking important financial indicators, including the net value of fixed assets and the year-end employment of enterprises; (2) exclude enterprise samples with less than 8 employees, because small enterprises with too few employees are more likely to misreport data; (3) exclude enterprise samples whose financial indicators are inconsistent with generally accepted accounting standards; (4) exclude enterprise samples established before 1949, because the enterprises established before 1949 are mainly Chinese medicine manufacturing enterprises, and it is difficult to know the age of these enterprises; (5) exclude enterprise samples whose intermediate input and wages payable to employees this year are less than 0; (6) exclude enterprise samples with fixed assets greater than their total assets, current assets greater than their total assets, current assets greater than their fixed assets, net value of fixed assets less than 0 , depreciation in this year greater than accumulated depreciation, and industrial added value greater than total output value; (7) exclude enterprise samples whose assets are not equal to corporate liabilities and owners' equity; (8) exclude enterprise samples whose water and various energy consumption are less than 0 ; (9) exclude enterprise samples whose discharge and removal of waste water, $\mathrm{SO}_{2}$, NOx, smoke dust, and industrial solid waste are missing values; (10) exclude enterprise samples above a designated size whose annual main business income is less than RMB 5 million. In addition, in view of the essential differences between enterprises in the waste resources and materials recovery processing industry, mining industry, power, heat, gas, and water production and supply industry, and ordinary industrial enterprises, refer to the practices of $\mathrm{Lu}$ and Tao [55], deleting the enterprise samples in the matching database with the industry code value of enterprises.

\subsection{Variables}

(1) Enterprises' green technology innovation

Considering the differences in industrial production and the availability of data, the measurement of the innovation behavior of enterprises' end-of-pipe technology innovation can be achieved via the removal rate of industrial wastewater, $\mathrm{SO}_{2}$, smoke, and dust and the comprehensive treatment rate of industrial solid waste. Industrial wastewater removal rate, $\mathrm{SO}_{2}$ removal rate, smoke dust removal rate, and the comprehensive treatment rate of industrial solid waste can be calculated by dividing the corresponding waste removal amount by the waste generation amount. The measurement of green process innovation is mainly based on energy use intensity, water use intensity, industrial wastewater discharge intensity, $\mathrm{SO}_{2}$ discharge intensity, smoke dust discharge intensity, and industrial solid waste discharge intensity. Due to the inherent differences in production processes of enterprises, the impact of the same market regulation on different green technology innovations may be quite different. Because of the data, this study does not consider green product innovation;

(2) Pollution charges 
Among many environmental regulation measures, pollution charges are a widely used and flexible method used for domestic environmental protection management at present, and these are also the main reasons for studying environmental regulations. The following mainly measures the intensity of environmental regulation by calculating the logarithm of the proportion of pollution charges to the total industrial output value. Figures 1-4 show the collection of pollution charges in China in 1998, 2002, 2007, and 2012, respectively. From the figures, the following conclusions can be roughly drawn: (1) the overall pollution charges show an upward trend in the time dimension; (2) in the spatial dimension, there are great differences in regional pollution charges - the pollution charges in the east are greater than those in the west, and those in the coastal areas are greater than those in the inland areas;

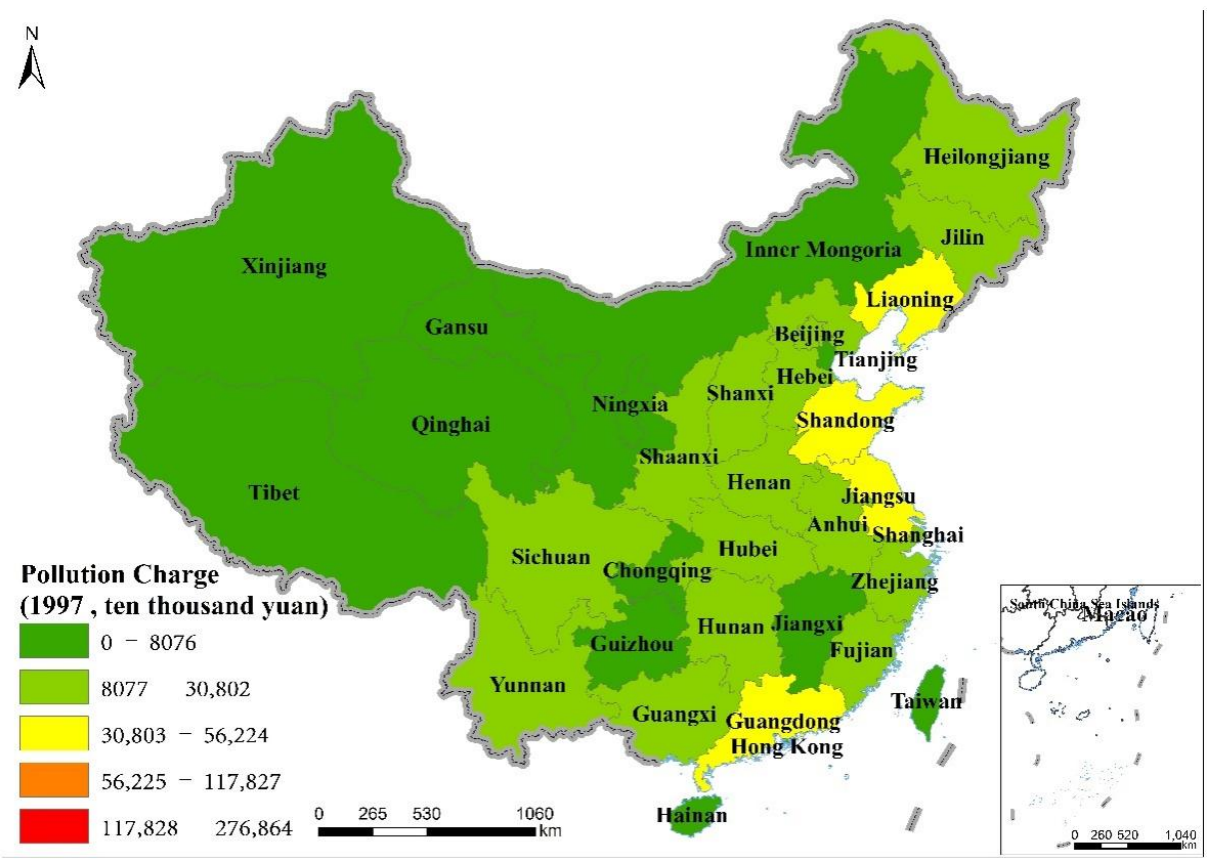

Figure 1. China's pollution charges in 1997.

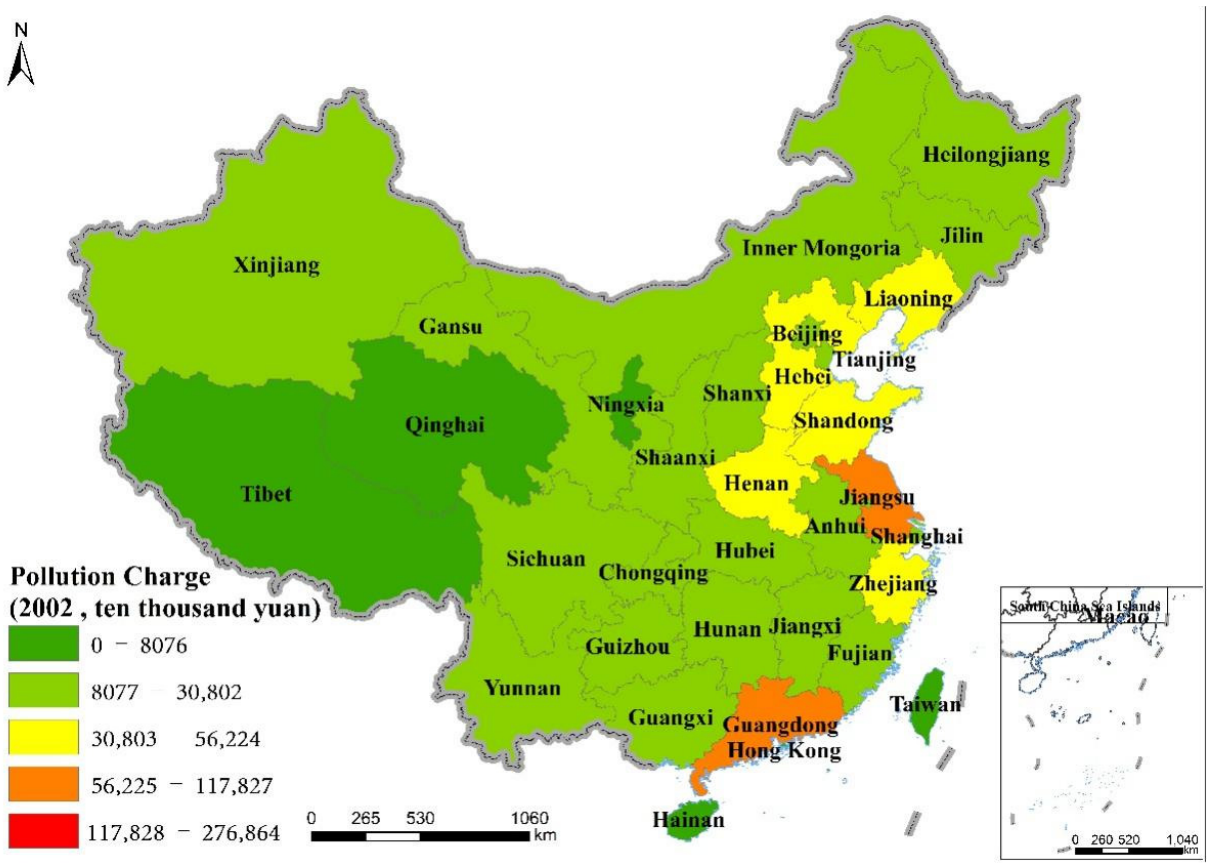

Figure 2. China's pollution charges in 2002. 


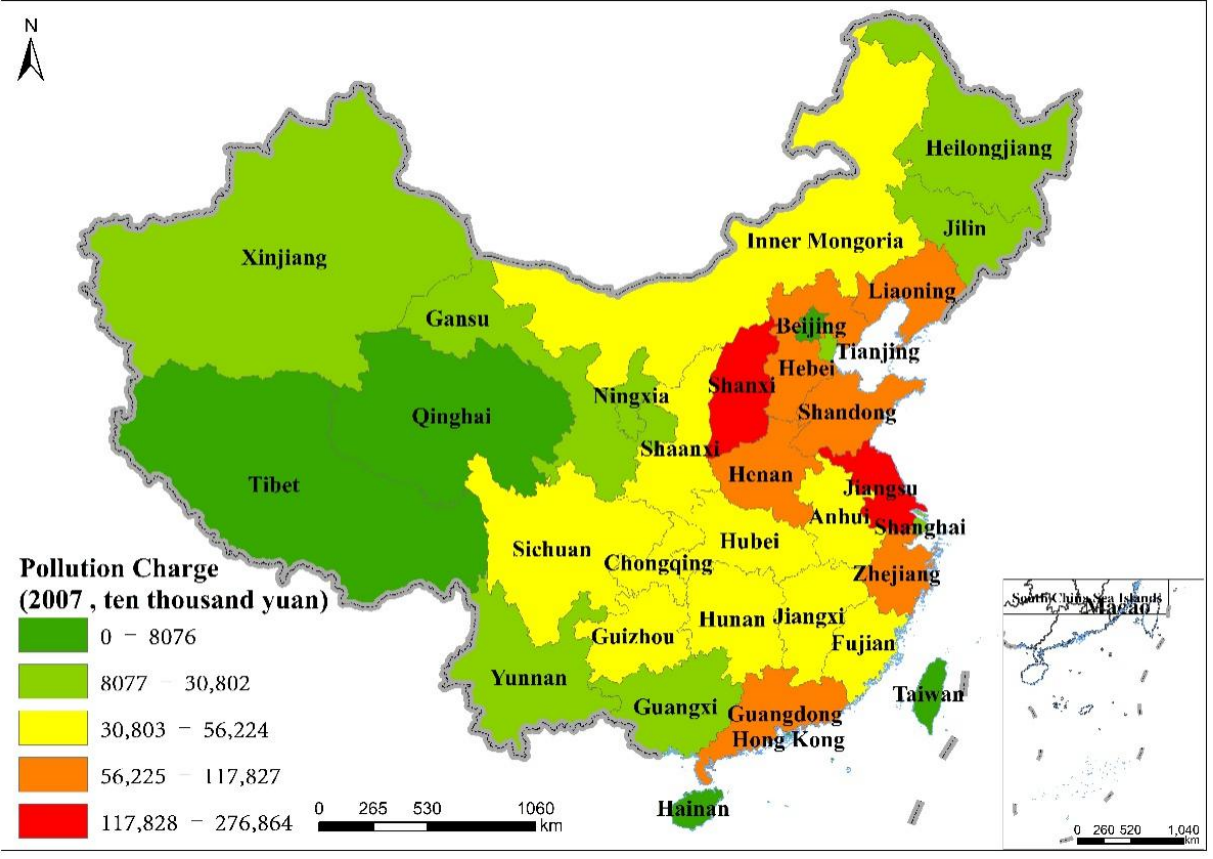

Figure 3. China's pollution charges in 2007.

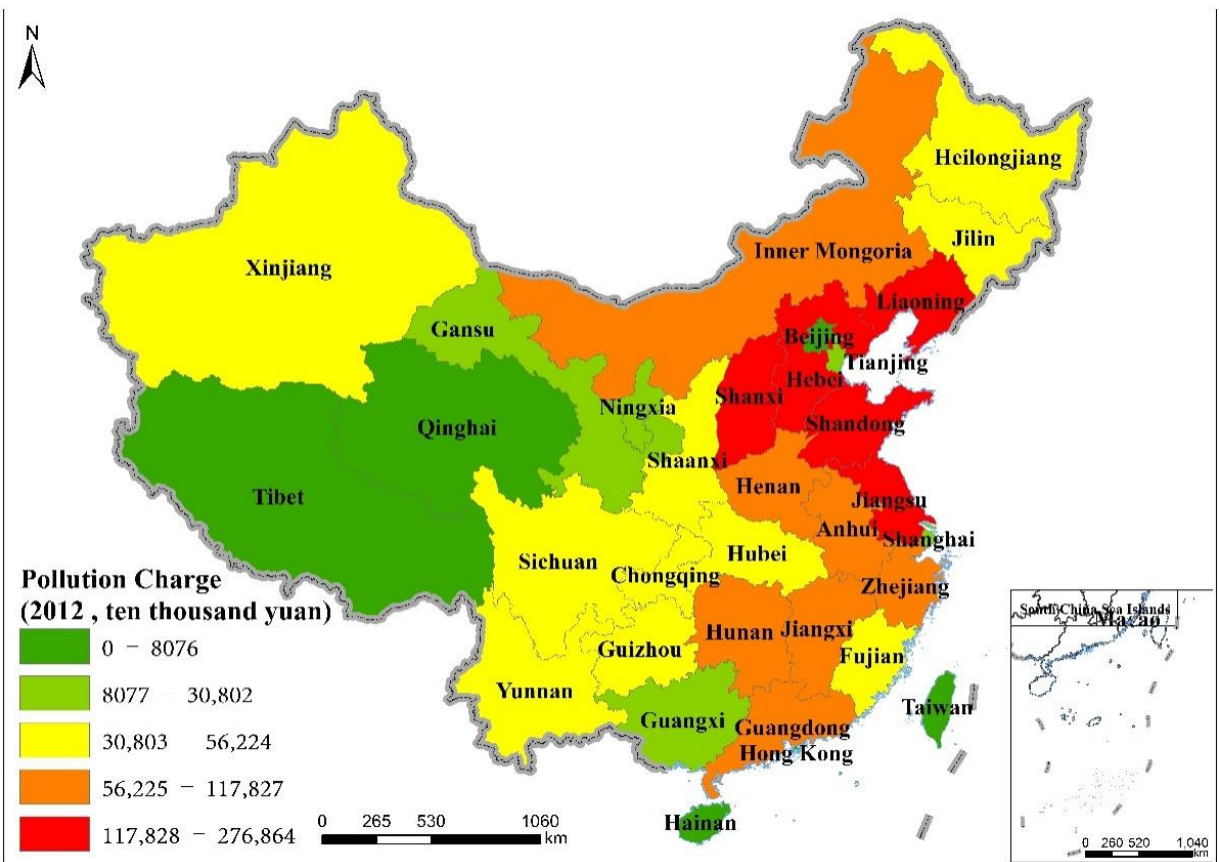

Figure 4. China's pollution charges in 2012.

(3) Control variables

In order to obtain estimation results as robust as possible, the control variables selected in this paper were as follows:

i. Industrial structure of the region where the enterprise is located: This article takes the proportion of regional industrial added value to total output value as the characteristic variable of industrial structure;

ii. ndustry that the enterprise belongs to: Considering that there are great differences in green technology innovation in different industries, it is necessary to consider the industrial characteristics of enterprises. Therefore, the industries to which 
the enterprises belong are divided into primary production industries, labor- and resource-intensive industries, and technology-intensive industries;

iii. Region where the enterprise is located: Enterprises located in different regions bear different regulation intensity, so it is necessary to control for the regions of enterprises in the model. We can also see from Figures 1-4 that there is a significant difference in pollution charges between the east and the west;

iv. In addition to the variables listed above, the estimation model in 2007 also controls the value-added tax payable, total assets, asset structure, number of employees, total capital, total profit, intermediate investment, enterprise age, annual normal production time, and employee structure. Due to the change in statistical caliber, the total industrial output value, VAT payable, income tax payable, accumulated depreciation, total assets, asset structure, total liabilities, total profits, enterprise scale, enterprise age, number of employees, and annual normal production time were controlled in the estimation model for 2012;

v. Among all of the variables, the intensity of emissions of $\mathrm{SO}_{2}$, smoke dust, industrial wastewater, and solid waste is measured by the logarithms of waste emission and total industrial output value quotient, Resource and energy consumption intensity are measured by the logarithms of resource and energy consumption and total industrial output value quotient, respectively. The waste removal rate is the logarithm of the actual removal rate of various waste products $\left(\mathrm{SO}_{2}\right.$, smoke dust, industrial wastewater, and solid waste), and the total industrial output value is controlled in the econometric model. Intermediate input, total industrial output value, interest expense, value-added tax payable, income tax payable, accumulated depreciation, total assets, total liabilities, total profits, enterprise age, number of employees, and annual normal production time are all logarithms of actual value. Enterprise age refers to the logarithm of survival time from opening to statistical node. The employee structure is the proportion of female employees to the total number of employees. Asset structure is the ratio of fixed assets to total assets.

\subsection{Model}

Because the current data are insufficient to construct panel data for empirical research, in order to achieve the research goal, this article mainly uses robust ordinary least squares (ROLS) to analyze the impact of pollution charges on different green technology innovations of enterprises. On the basis of previous studies, the quadratic term of pollution charge is introduced into the econometric model to further demonstrate the nonlinear relationship between pollution charges and enterprise green technology innovation, as shown in Equation (1).

$$
y_{i}=\alpha+\beta_{1} P C_{i}+\beta_{2} P C_{i}^{2}+\sum \gamma_{i} \text { Cont }_{i}+\varepsilon_{i}
$$

where $y_{i}$ is the green technology innovation of the enterprise, including end-pipe green technology innovation (measured by industrial wastewater removal intensity, $\mathrm{SO}_{2}$ removal intensity, smoke and dust removal intensity, and comprehensive utilization intensity of industrial solid waste) and green process innovation (measured by energy use intensity, water resource use intensity, industrial wastewater discharge intensity, $\mathrm{SO}_{2}$ emission intensity, smoke and dust emission intensity, and industrial solid waste emission intensity). $P C_{i}{ }^{2}$ and $P C_{i}$ are the square terms of the intensity of pollution charge and the intensity of pollution charge, respectively. Cont $t_{i}$ represents control variables, $\varepsilon_{i}$ is a random error term, and $\alpha$ is an intercept item.

\section{Results}

\subsection{Descriptive Statistics}

The mean value, standard deviation, maximum value, and minimum value of the main variables in 2012 are shown in Table 1, and the results in 2007 are similar, so they are not listed. 
Table 1. Descriptive statistical results of the main variables for 2012.

\begin{tabular}{|c|c|c|c|c|c|}
\hline Variables & Samples & Means & SD & Min & Max \\
\hline $\mathrm{SO}_{2}$ emission intensity & 10,001 & -9.311 & 2.035 & -21.379 & -1.786 \\
\hline Smoke and dust emission intensity & 10,001 & -8.356 & 2.352 & -20.700 & 0.736 \\
\hline Solid waste discharge intensity & 10,001 & -5.894 & 2.019 & -14.851 & 1.920 \\
\hline Wastewater discharge intensity & 10,001 & -1.232 & 2.139 & -13.226 & 4.888 \\
\hline $\mathrm{SO}_{2}$ removal intensity & 10,001 & -9.014 & 0.599 & -9.210 & -1.771 \\
\hline Smoke and dust removal intensity & 10,001 & -7.771 & 1.505 & -9.210 & 0.735 \\
\hline Solid waste removal intensity & 10,001 & -5.821 & 1.790 & -9.210 & 1.272 \\
\hline Wastewater removal intensity & 10,001 & -1.346 & 2.597 & -9.210 & 5.420 \\
\hline Gross industrial output value & 10,001 & 12.327 & 1.343 & 9.916 & 16.778 \\
\hline Pollution charges & 10,030 & -8.051 & 0.444 & -9.270 & -6.787 \\
\hline VAT payable & 9462 & 8.418 & 1.746 & 2.944 & 13.352 \\
\hline Income tax payable & 6602 & 7.310 & 2.041 & 1.386 & 12.276 \\
\hline Accumulated depreciation & 9833 & 9.713 & 1.895 & 4.575 & 15.222 \\
\hline Total Assets & 10,029 & 11.700 & 1.563 & 6.738 & 18.925 \\
\hline Asset structure & 9943 & 0.378 & 0.219 & 0.011 & 0.954 \\
\hline Total liabilities & 10,003 & 10.828 & 1.811 & 5.553 & 16.036 \\
\hline Total profit & 9999 & 9.138 & 1.940 & 3.296 & 13.838 \\
\hline Enterprise scale & 10,030 & 2.493 & 0.647 & 1.000 & 4.000 \\
\hline Enterprise age & 10,011 & 12.872 & 10.616 & 1.000 & 58.000 \\
\hline Annual production time & 10,030 & 8.363 & 0.618 & 5.704 & 9.078 \\
\hline Technology-intensive enterprise & 10,030 & 0.487 & 0.500 & 0.000 & 1.000 \\
\hline Enterprise in the eastern provinces & 10,030 & 0.606 & 0.489 & 0.000 & 1.000 \\
\hline
\end{tabular}

By analyzing the correlation between pollution charges and enterprise green technology innovation in 2012, in Table 2, it can be seen that (1) the pollution charges in 2012 had a significant positive correlation with $\mathrm{SO}_{2}$ emission intensity, smoke dust emission intensity, and $\mathrm{SO}_{2}$ removal intensity at the $1 \%$ confidence level, and the correlation coefficients were $0.040,0.030$, and 0.073 , respectively; (2) the pollution charges were negatively correlated with the discharge intensity and removal intensity of industrial wastewater at the confidence level of $1 \%$, and the correlation coefficients are -0.045 and -0.054 , respectively; and (3) pollution charges are negatively correlated with solid waste emission intensity, positively correlated with smoke and dust removal intensity, and negatively correlated with solid waste removal intensity, but the correlation coefficient is not significant at the $10 \%$ confidence level. The above results show that, except for smoke dust and solid waste, the pollution charges promote the end-treatment of $\mathrm{SO}_{2}$, but inhibit the improvement of the $\mathrm{SO}_{2}$ emission reduction process. However, the effect of pollution charges on industrial wastewater is the complete oppositethat is, pollution charges inhibit the end-treatment of industrial wastewater, but promote the improvement of the industrial wastewater removal process.

\subsection{Empirical Test Results for 2012}

Pollution charges at a given time can have a certain influence on different types of green technology innovation of enterprises. (1) From Table 3, it can be seen that pollution charges are positively correlated with $\mathrm{SO}_{2}$ emission intensity, smoke dust emission intensity, and solid waste emission intensity at the $1 \%$ confidence level, with regression coefficients of $0.357,0.293$, and 0.162 , respectively; that is, when increasing pollution charges in total industrial output value, $\mathrm{SO}_{2}$ emission intensity, smoke dust emission intensity, and solid waste emission intensity increase correspondingly. There is a significant negative correlation between pollution charges and industrial wastewater discharge intensity at the confidence level of $5 \%$, and the regression coefficient is -0.481 . (2) From Table 4, it can be seen that the pollution charges are significantly positively correlated with the $\mathrm{SO}_{2}$ removal intensity and solid waste removal intensity at the confidence levels of $1 \%$ and $10 \%$, respectively, and the regression coefficients are 0.099 and 0.085 , respectively. There is a significant negative correlation between pollution charges and industrial wastewater removal intensity at the confidence level of $1 \%$, and the regression coefficient is -0.304 . The regression coefficient between industrial pollution cost and smoke and dust removal intensity is not significant. (3) The results given in Table 5 show that the industrial pollution charges are negatively correlated with the total energy intensity, power consumption intensity, and fuel oil consumption intensity at the confidence 
levels of $1 \%, 1 \%$, and $5 \%$ respectively, and the regression coefficients are $-2.22,-0.428$, and -0.339 , respectively. Pollution charges are positively correlated with coal consumption intensity and natural gas consumption intensity at the confidence level of $1 \%$, with regression coefficients of 0.555 and 0.378 , respectively.

Table 2. Correlation coefficient matrix of major variables in 2012.

\begin{tabular}{|c|c|c|c|c|c|c|c|c|c|}
\hline Variables & 1 & 2 & 3 & 4 & 5 & 6 & 7 & 8 & 9 \\
\hline $\mathrm{SO}_{2}$ emission intensity & 1.000 & & & & & & & & \\
\hline $\begin{array}{l}\text { Smoke and dust emission } \\
\text { intensity }\end{array}$ & $0.740^{* * *}$ & 1.000 & & & & & & & \\
\hline $\begin{array}{l}\text { Solid waste discharge } \\
\text { intensity }\end{array}$ & $0.675^{* * *}$ & $0.659 * * *$ & 1.000 & & & & & & \\
\hline $\begin{array}{l}\text { Wastewater discharge } \\
\text { intensity }\end{array}$ & $0.473^{* * *}$ & $0.368^{* * *}$ & $0.432 * * *$ & 1.000 & & & & & \\
\hline $\mathrm{SO}_{2}$ removal intensity & $0.225^{* * *}$ & $0.277^{* * *}$ & $0.273 * * *$ & $0.156^{* * *}$ & 1.000 & & & & \\
\hline $\begin{array}{l}\text { Smoke and dust removal } \\
\text { intensity }\end{array}$ & $0.575^{* * *}$ & $0.860 * * *$ & $0.584^{* * *}$ & $0.299 * * *$ & $0.322 * * *$ & 1.000 & & & \\
\hline Solid waste removal intensity & $0.663 * * *$ & $0.653 * * *$ & $0.957 * * *$ & $0.426^{* * *}$ & $0.275^{* * *}$ & $0.604^{* * *}$ & 1.000 & & \\
\hline Wastewater removal intensity & $0.402 * * *$ & $0.347^{* * *}$ & $0.400 * * *$ & $0.761^{* * *}$ & $0.168^{* * *}$ & $0.337^{* * *}$ & $0.396^{* * *}$ & 1.000 & \\
\hline Pollution charges & $0.040 * * *$ & $0.030 * * *$ & -0.001 & $-0.045^{* * *}$ & $0.073^{* * *}$ & 0.008 & -0.010 & $-0.054^{* * *}$ & 1.000 \\
\hline
\end{tabular}

*** $p<0.01$.

Table 3. Regression results of the impact of pollution charges on enterprises' process improvement and technological innovation in 2012.

\begin{tabular}{|c|c|c|c|c|}
\hline & $\begin{array}{c}\mathrm{SO}_{2} \text { Emission Intensity } \\
\mathrm{M} 1\end{array}$ & $\begin{array}{c}\text { Smoke and Dust } \\
\text { Emission Intensity M2 }\end{array}$ & $\begin{array}{c}\text { Solid Waste Discharge } \\
\text { Intensity M3 }\end{array}$ & $\begin{array}{l}\text { Industrial Water } \\
\text { Intensity M4 }\end{array}$ \\
\hline Pollution charges & $\begin{array}{c}0.357 * * * \\
(6.67)\end{array}$ & $\begin{array}{l}0.293 \\
(4.43)\end{array}$ & $\begin{array}{l}0.162 * \\
(2.99)\end{array}$ & $\begin{array}{l}-0481 \\
(-2.15)\end{array}$ \\
\hline Square of pollution charge & $\begin{array}{l}0.005 \\
(0.26)\end{array}$ & $\begin{array}{l}0.006 \\
(0.35)\end{array}$ & $\begin{array}{l}-0.001 \\
(-1.02)\end{array}$ & $\begin{array}{c}0.196 * \\
(1.85)\end{array}$ \\
\hline Regional industrial structure & $\begin{array}{l}-1.561^{* * *} \\
(-2.01)\end{array}$ & $\begin{array}{l}-0.665 \\
(-0.69)\end{array}$ & $\begin{array}{l}-3.529 * * * \\
(-4.49)\end{array}$ & $\begin{array}{l}1.811^{* * *} \\
(-2.26)\end{array}$ \\
\hline VAT payable & $\begin{array}{c}0.0511^{* *} \\
(2.56)\end{array}$ & $\begin{array}{l}0.0789 * * * \\
(3.20)\end{array}$ & $\begin{array}{l}0.0305 \\
(1.51)\end{array}$ & $\begin{array}{c}0.06113^{* * *} \\
(3.05)\end{array}$ \\
\hline Income tax payable & $\begin{array}{l}-0.0222 \\
(-1.09)\end{array}$ & $\begin{array}{l}-0.0377 \\
(-1.51)\end{array}$ & $\begin{array}{c}-0.0374 * \\
(-1.82)\end{array}$ & $\begin{array}{c}-0.0446^{* *} \\
(-2.18)\end{array}$ \\
\hline Accumulated depreciation & $\begin{array}{c}0.0947^{* * * *} \\
(4.52)\end{array}$ & $\begin{array}{c}0.143^{* * *} \\
(5.52)\end{array}$ & $\begin{array}{c}0.178^{* * *} \\
(8.40)\end{array}$ & $\begin{array}{c}0.116^{* * *} \\
(5.51)\end{array}$ \\
\hline Gross industrial output value & $\begin{array}{l}-0.959 * * * \\
(-26.27)\end{array}$ & $\begin{array}{l}-1.006^{* * *} \\
(-22.32)\end{array}$ & $\begin{array}{l}-0.909 * * * \\
(-24.58)\end{array}$ & $\begin{array}{l}-0.965^{* * *} \\
(-26.22)\end{array}$ \\
\hline Asset structure & $\begin{array}{c}0.985^{* * *} \\
(8.65)\end{array}$ & $\begin{array}{c}1.233^{* * * *} \\
(8.78)\end{array}$ & $\begin{array}{l}0.908^{* * *} \\
(7.88)\end{array}$ & $\begin{array}{c}0.520^{* * * *} \\
(4.53)\end{array}$ \\
\hline Total liabilities & $\begin{array}{c}0.141^{* * *} \\
(6.44)\end{array}$ & $\begin{array}{l}0.210 * * * \\
(7.79)\end{array}$ & $\begin{array}{c}0.185^{* * *} \\
(8.35)\end{array}$ & $\begin{array}{c}0.131^{* * *} \\
(5.95)\end{array}$ \\
\hline Total profit & $\begin{array}{l}-0.0344 \\
(-1.37)\end{array}$ & $\begin{array}{l}-0.0294 \\
(-0.95)\end{array}$ & $\begin{array}{l}-0.0275 \\
(-1.08)\end{array}$ & $\begin{array}{c}0.00257 \\
(0.10)\end{array}$ \\
\hline Enterprise scale & $\begin{array}{l}-0.207 \\
(-0.99)\end{array}$ & $\begin{array}{c}-1.202 * * * \\
(-4.64)\end{array}$ & $\begin{array}{c}-1.379 * * * \\
(-6.48)\end{array}$ & $\begin{array}{l}-0.585^{* *} \\
(-2.76)\end{array}$ \\
\hline Enterprise age & $\begin{array}{l}-0.00465 \\
(-0.77)\end{array}$ & $\begin{array}{l}-0.0106 \\
(-1.42)\end{array}$ & $\begin{array}{l}-0.00147 \\
(-0.24)\end{array}$ & $\begin{array}{l}0.0283^{* * *} \\
(4.63)\end{array}$ \\
\hline Enterprise employment & $\begin{array}{c}0.0662^{* * *} \\
(2.65)\end{array}$ & $\begin{array}{c}0.0699 * * \\
(2.27)\end{array}$ & $\begin{array}{c}0.0875^{* * *} \\
(3.46)\end{array}$ & $\begin{array}{c}0.0695^{* * *} \\
(2.76)\end{array}$ \\
\hline Annual normal production time & $\begin{array}{c}1.199 * * * \\
(31.91)\end{array}$ & $\begin{array}{c}1.272 * * * \\
(27.41)\end{array}$ & $\begin{array}{c}1.076^{* * *} \\
(28.25)\end{array}$ & $\begin{array}{c}0.956^{* * *} \\
(25.22)\end{array}$ \\
\hline Technology-intensive enterprise & $\begin{array}{c}-0.388^{* * *} \\
(-8.83)\end{array}$ & $\begin{array}{c}-0.304^{* * *} \\
(-5.61)\end{array}$ & $\begin{array}{c}-0.323 * * * \\
(-7.27)\end{array}$ & $\begin{array}{c}-1.457^{* * * *} \\
(-32.92)\end{array}$ \\
\hline Enterprise in the eastern provinces & $\begin{array}{l}-0.0692 \\
(-1.45)\end{array}$ & $\begin{array}{c}-0.352 * * * \\
(-5.98)\end{array}$ & $\begin{array}{c}-0.284^{* * *} \\
(-5.86)\end{array}$ & $\begin{array}{l}-0.0532 \\
(-1.10)\end{array}$ \\
\hline$N$ & 3115 & 3119 & 3680 & 3963 \\
\hline$R^{2}$ & 0.265 & 0.250 & 0.225 & 0.189 \\
\hline
\end{tabular}

$t$-Statistics in parentheses; ${ }^{*} p<0.1,{ }^{* *} p<0.05,{ }^{* * *} p<0.01$. 
Table 4. Regression results of the impact of pollution charges on enterprises' end-treatment technological innovation in 2012.

\begin{tabular}{|c|c|c|c|c|}
\hline & $\begin{array}{c}\mathrm{SO}_{2} \text { Removal } \\
\text { Intensity M5 }\end{array}$ & $\begin{array}{c}\text { Smoke and } \\
\text { Dust Removal } \\
\text { Intensity M6 }\end{array}$ & $\begin{array}{l}\text { Solid Waste } \\
\text { Removal } \\
\text { Intensity M7 }\end{array}$ & $\begin{array}{c}\text { Wastewater } \\
\text { Removal } \\
\text { Intensity M8 }\end{array}$ \\
\hline Pollution charges & $\begin{array}{l}0.0991 * * * \\
(5.51)\end{array}$ & $\begin{array}{l}0.0658 \\
(1.52)\end{array}$ & $\begin{array}{c}0.0852 * \\
(1.76)\end{array}$ & $\begin{array}{l}-0.304^{* * *} \\
(-4.13)\end{array}$ \\
\hline $\begin{array}{l}\text { Square of } \\
\text { pollution charge }\end{array}$ & $\begin{array}{l}-0.075 \\
(-1.09)\end{array}$ & $\begin{array}{l}-0.398 \\
(-0.20)\end{array}$ & $\begin{array}{l}-1.562 \\
(-0.82)\end{array}$ & $\begin{array}{l}1.023 \\
(0.59)\end{array}$ \\
\hline $\begin{array}{l}\text { Regional } \\
\text { industrial } \\
\text { structure }\end{array}$ & $\begin{array}{c}-1.024^{* * *} \\
(-3.93)\end{array}$ & $\begin{array}{l}-0.0156 \\
(-0.02)\end{array}$ & $\begin{array}{c}-2.917^{* * *} \\
(-4.15)\end{array}$ & $\begin{array}{c}5.449 * * * \\
(5.11)\end{array}$ \\
\hline VAT payable & $\begin{array}{c}-0.00235 \\
(-0.35)\end{array}$ & $\begin{array}{c}0.0531^{* *} \\
(3.28)\end{array}$ & $\begin{array}{c}0.0381 * * \\
(2.11)\end{array}$ & $\begin{array}{c}0.0716^{* * *} \\
(2.61)\end{array}$ \\
\hline $\begin{array}{l}\text { Income tax } \\
\text { payable }\end{array}$ & $\begin{array}{c}-0.00354 \\
(-0.52)\end{array}$ & $\begin{array}{l}-0.0124 \\
(-0.75)\end{array}$ & $\begin{array}{c}-0.0390 * * \\
(-2.12)\end{array}$ & $\begin{array}{l}0.0281 \\
(1.01)\end{array}$ \\
\hline $\begin{array}{l}\text { Accumulated } \\
\text { depreciation }\end{array}$ & $\begin{array}{c}0.0364^{* * *} \\
(5.19)\end{array}$ & $\begin{array}{c}0.131^{* * *} \\
(7.71)\end{array}$ & $\begin{array}{c}0.146^{* * *} \\
(7.69)\end{array}$ & $\begin{array}{c}0.156^{* * *} \\
(5.42)\end{array}$ \\
\hline $\begin{array}{l}\text { Gross industrial } \\
\text { output value }\end{array}$ & $\begin{array}{c}-0.0460 * * * \\
(-3.75)\end{array}$ & $\begin{array}{l}-0.565^{* * *} \\
(-19.11)\end{array}$ & $\begin{array}{l}-0.797^{* * *} \\
(-24.10)\end{array}$ & $\begin{array}{l}-0.882^{* * *} \\
(-17.59)\end{array}$ \\
\hline Asset structure & $\begin{array}{c}0.188^{* * *} \\
(4.92)\end{array}$ & $\begin{array}{c}0.921^{* * *} \\
(10.00)\end{array}$ & $\begin{array}{l}0.950 * * * \\
(9.22)\end{array}$ & $\begin{array}{l}0.784^{* * *} \\
(5.02)\end{array}$ \\
\hline Total liabilities & $\begin{array}{l}0.0421^{* * *} \\
(5.74)\end{array}$ & $\begin{array}{c}0.141^{* * *} \\
(7.96)\end{array}$ & $\begin{array}{c}0.173^{* * *} \\
(8.76)\end{array}$ & $\begin{array}{c}0.125^{* * *} \\
(4.17)\end{array}$ \\
\hline Total profit & $\begin{array}{c}-0.0149 * \\
(-1.76)\end{array}$ & $\begin{array}{l}-0.0143 \\
(-0.70)\end{array}$ & $\begin{array}{l}-0.0193 \\
(-0.85)\end{array}$ & $\begin{array}{l}0.0824^{* *} \\
(-2.38)\end{array}$ \\
\hline Enterprise scale & $\begin{array}{c}-0.207^{* * *} \\
(-2.94)\end{array}$ & $\begin{array}{l}-1.016^{* * *} \\
(-5.97)\end{array}$ & $\begin{array}{l}-1.262^{* * *} \\
(-6.63)\end{array}$ & $\begin{array}{l}-0.342 \\
(-1.19)\end{array}$ \\
\hline Enterprise age & $\begin{array}{c}-0.000678 \\
(-0.77)\end{array}$ & $\begin{array}{l}-0.0106 \\
(-1.42)\end{array}$ & $\begin{array}{c}-0.00147 \\
(-0.24)\end{array}$ & $\begin{array}{c}0.0283^{* * * *} \\
(4.63)\end{array}$ \\
\hline $\begin{array}{l}\text { Enterprise } \\
\text { employment }\end{array}$ & $\begin{array}{c}0.00303 \\
(0.36)\end{array}$ & $\begin{array}{c}0.0475^{* *} \\
(2.35)\end{array}$ & $\begin{array}{c}0.0905^{* * *} \\
(4.00)\end{array}$ & $\begin{array}{c}0.143^{* * *} \\
(4.17)\end{array}$ \\
\hline $\begin{array}{l}\text { Annual normal } \\
\text { production time }\end{array}$ & $\begin{array}{c}0.145^{* * *} \\
(11.50)\end{array}$ & $\begin{array}{l}0.754^{* * *} \\
(24.77)\end{array}$ & $\begin{array}{c}0.946^{* * *} \\
(27.79)\end{array}$ & $\begin{array}{l}1.090^{* * *} \\
(21.11)\end{array}$ \\
\hline $\begin{array}{l}\text { Technology- } \\
\text { intensive } \\
\text { enterprise }\end{array}$ & $\begin{array}{c}-0.0358^{* *} \\
(-2.43)\end{array}$ & $\begin{array}{c}-0.147^{* * * *} \\
(-4.13)\end{array}$ & $\begin{array}{c}-0.305^{* * *} \\
(-7.68)\end{array}$ & $\begin{array}{c}-1.387^{* * *} \\
(-22.99)\end{array}$ \\
\hline $\begin{array}{l}\text { Enterprise in the } \\
\text { eastern provinces }\end{array}$ & $\begin{array}{c}-0.0358^{* *} \\
(-2.24)\end{array}$ & $\begin{array}{c}-0.227^{* * *} \\
(-5.86)\end{array}$ & $\begin{array}{c}-0.279 * * * \\
(-6.45)\end{array}$ & $\begin{array}{c}-0.208^{* * *} \\
(-3.17)\end{array}$ \\
\hline $\begin{array}{l}N \\
R^{2}\end{array}$ & $\begin{array}{l}6283 \\
0.282\end{array}$ & $\begin{array}{l}6283 \\
0.197\end{array}$ & $\begin{array}{l}6283 \\
0.262\end{array}$ & $\begin{array}{l}6283 \\
0.232\end{array}$ \\
\hline
\end{tabular}


Table 5. Regression results of the impact of pollution charges on enterprises' energy-saving technological innovation in 2012.

\begin{tabular}{|c|c|c|c|c|c|}
\hline & $\begin{array}{l}\text { Energy Con- } \\
\text { sumption } \\
\text { Intensity M9 }\end{array}$ & $\begin{array}{l}\text { Enterprise } \\
\text { Power } \\
\text { Intensity } \\
\text { M10 }\end{array}$ & $\begin{array}{l}\text { Enterprise } \\
\text { Coal } \\
\text { Intensity } \\
\text { M11 }\end{array}$ & $\begin{array}{c}\text { Enterprise } \\
\text { Natural Gas } \\
\text { Intensity } \\
\text { M12 }\end{array}$ & $\begin{array}{c}\text { Enterprise } \\
\text { Fuel Oil } \\
\text { Intensity } \\
\text { M13 }\end{array}$ \\
\hline $\begin{array}{l}\text { Pollution } \\
\text { charges }\end{array}$ & $\begin{array}{c}-2.222 * * * \\
(-3.06)\end{array}$ & $\begin{array}{c}-0.428^{* * *} \\
(-3.72)\end{array}$ & $\begin{array}{c}0.555^{* * *} \\
(5.57)\end{array}$ & $\begin{array}{c}0.378^{* * *} \\
(3.46)\end{array}$ & $\begin{array}{l}-0.339 * * \\
(-2.07)\end{array}$ \\
\hline $\begin{array}{l}\text { Square of } \\
\text { pollution } \\
\text { charge }\end{array}$ & $\begin{array}{c}1.198^{* * *} \\
(3.40)\end{array}$ & $\begin{array}{c}0.235^{* * *} \\
(4.22)\end{array}$ & $\begin{array}{l}-1.036 \\
(-0.38)\end{array}$ & $\begin{array}{l}-0.956 \\
(-1.01)\end{array}$ & $\begin{array}{l}0.159 \\
(0.36)\end{array}$ \\
\hline $\begin{array}{l}\text { Regional } \\
\text { industrial } \\
\text { structure }\end{array}$ & $\begin{array}{l}-1.558 \\
(-1.36)\end{array}$ & $\begin{array}{c}-2.621^{* * *} \\
(-6.18)\end{array}$ & $\begin{array}{l}-1.692 \\
(-1.59)\end{array}$ & $\begin{array}{l}-1.798 \\
(-1.58)\end{array}$ & $\begin{array}{l}2.650 \\
(1.48)\end{array}$ \\
\hline VAT payable & $\begin{array}{c}0.0507 \\
(1.32)\end{array}$ & $\begin{array}{c}0.0137 \\
(1.24)\end{array}$ & $\begin{array}{c}0.0506 \\
(1.35)\end{array}$ & $\begin{array}{c}0.0913 \text { * } \\
(2.48)\end{array}$ & $\begin{array}{c}0.0539 \\
(1.02)\end{array}$ \\
\hline $\begin{array}{l}\text { Income tax } \\
\text { payable }\end{array}$ & $\begin{array}{l}-0.332 * \\
(-1.80)\end{array}$ & $\begin{array}{l}-0.122 * \\
(-1.93)\end{array}$ & $\begin{array}{l}-0.356^{* *} \\
(-2.06)\end{array}$ & $\begin{array}{l}-0.0444 \\
(-0.16)\end{array}$ & $\begin{array}{l}0.567 \\
(1.42)\end{array}$ \\
\hline $\begin{array}{l}\text { Accumulated } \\
\text { depreciation }\end{array}$ & $\begin{array}{c}0.193^{* * *} \\
(4.99)\end{array}$ & $\begin{array}{c}0.220 * * * \\
(18.80)\end{array}$ & $\begin{array}{c}0.220^{* * *} \\
(6.13)\end{array}$ & $\begin{array}{l}0.0409 \\
(0.86)\end{array}$ & $\begin{array}{c}0.161^{* *} \\
(2.40)\end{array}$ \\
\hline $\begin{array}{l}\text { Gross } \\
\text { industrial } \\
\text { output value }\end{array}$ & $\begin{array}{l}-0.909^{* * *} \\
(-12.91)\end{array}$ & $\begin{array}{c}-0.947^{* * *} \\
(-45.71)\end{array}$ & $\begin{array}{l}-0.692^{* * *} \\
(-10.51)\end{array}$ & $\begin{array}{l}-0.861^{* * *} \\
(-11.57)\end{array}$ & $\begin{array}{c}-0.666^{* * *} \\
(-5.89)\end{array}$ \\
\hline Asset structure & $\begin{array}{c}1.318^{* * *} \\
(6.38)\end{array}$ & $\begin{array}{c}0.521 * * * \\
(8.14)\end{array}$ & $\begin{array}{c}0.895^{* * *} \\
(4.69)\end{array}$ & $\begin{array}{c}1.152^{* * *} \\
(4.37)\end{array}$ & $\begin{array}{l}-0.231 \\
(-0.59)\end{array}$ \\
\hline Total liabilities & $\begin{array}{c}0.191^{* * *} \\
(4.50)\end{array}$ & $\begin{array}{c}0.258^{* * *} \\
(21.14)\end{array}$ & $\begin{array}{c}0.195^{* * *} \\
(4.98)\end{array}$ & $\begin{array}{c}0.0873 \text { * } \\
(1.73)\end{array}$ & $\begin{array}{l}-0.0578 \\
(-0.76)\end{array}$ \\
\hline Total profit & $\begin{array}{c}-0.101^{* * *} \\
(-2.41)\end{array}$ & $\begin{array}{c}-0.0556^{* * *} \\
(-3.97)\end{array}$ & $\begin{array}{c}-0.157^{* * *} \\
(-4.12)\end{array}$ & $\begin{array}{c}-0.0613 \\
(-1.18)\end{array}$ & $\begin{array}{l}-0.0666 \\
(-0.85)\end{array}$ \\
\hline $\begin{array}{l}\text { Enterprise } \\
\text { scale }\end{array}$ & $\begin{array}{c}-1.623^{* * *} \\
(-4.14)\end{array}$ & $\begin{array}{c}-0.350 * * * \\
(-3.01)\end{array}$ & $\begin{array}{c}-1.261^{* * *} \\
(-3.57)\end{array}$ & $\begin{array}{l}-0.319 \\
(-0.90)\end{array}$ & $\begin{array}{c}0.109 \\
(-0.21)\end{array}$ \\
\hline Enterprise age & $\begin{array}{c}-0.0246^{* *} \\
(-2.18)\end{array}$ & $\begin{array}{c}-0.00845^{* *} \\
(-2.35)\end{array}$ & $\begin{array}{c}-0.0212 * \\
(1.99)\end{array}$ & $\begin{array}{c}0.00258 \\
(0.21)\end{array}$ & $\begin{array}{c}-0.0361 \\
(-1.93)\end{array}$ \\
\hline $\begin{array}{l}\text { Enterprise } \\
\text { employment }\end{array}$ & $\begin{array}{l}0.0681 \\
(1.41)\end{array}$ & $\begin{array}{c}0.116^{* * *} \\
(7.88)\end{array}$ & $\begin{array}{c}0.120 * * \\
(2.70)\end{array}$ & $\begin{array}{l}0.0233 \\
(0.56)\end{array}$ & $\begin{array}{l}-0.0331 \\
(-0.51)\end{array}$ \\
\hline $\begin{array}{l}\text { Annual normal } \\
\text { production } \\
\text { time }\end{array}$ & $\begin{array}{c}1.186^{* * *} \\
(17.10)\end{array}$ & $\begin{array}{c}0.984^{* * *} \\
(47.48)\end{array}$ & $\begin{array}{c}1.187^{* * *} \\
(17.88)\end{array}$ & $\begin{array}{c}1.328^{* * *} \\
(17.26)\end{array}$ & $\begin{array}{c}0.708^{* * *} \\
(6.00)\end{array}$ \\
\hline $\begin{array}{l}\text { Technology- } \\
\text { intensive } \\
\text { enterprise }\end{array}$ & $\begin{array}{c}-0.00822 \\
(-0.10)\end{array}$ & $\begin{array}{c}0.330^{* * *} \\
(12.64)\end{array}$ & $\begin{array}{c}0.234^{* *} \\
(2.95)\end{array}$ & $\begin{array}{c}0.337^{* * *} \\
(2.96)\end{array}$ & $\begin{array}{l}0.173 \\
(1.11)\end{array}$ \\
\hline $\begin{array}{l}\text { Enterprise in } \\
\text { the eastern } \\
\text { provinces }\end{array}$ & $\begin{array}{c}-0.327^{* * *} \\
(-3.78)\end{array}$ & $\begin{array}{c}0.295^{* * *} \\
(10.76)\end{array}$ & $\begin{array}{c}-0.210^{* *} \\
(-2.62)\end{array}$ & $\begin{array}{l}-0.110 \\
(-1.11)\end{array}$ & $\begin{array}{c}0.336^{* *} \\
(2.01)\end{array}$ \\
\hline $\begin{array}{l}N \\
R^{2}\end{array}$ & $\begin{array}{l}1674 \\
0.310\end{array}$ & $\begin{array}{l}18334 \\
0.304\end{array}$ & $\begin{array}{l}1739 \\
0.315\end{array}$ & $\begin{array}{l}1720 \\
0.314\end{array}$ & $\begin{array}{c}870 \\
0.170\end{array}$ \\
\hline
\end{tabular}

$t$-Statistics in parentheses; ${ }^{*} p<0.1,{ }^{* *} p<0.05,{ }^{* * *} p<0.01$. 
The influence of pollution charges on different types of green technology innovation at a given time is inconsistent. (1) Pollution charges can improve the emission intensity of $\mathrm{SO}_{2}$, smoke and dust, and solid waste, but reduce the emission intensity of industrial wastewater. Pollution charges can promote the intensity of $\mathrm{SO}_{2}$ removal and solid waste discharge, but reduce the intensity of industrial wastewater removal. Pollution charges have a restraining effect on total energy intensity, power consumption intensity, and fuel oil consumption intensity, but they significantly increase coal consumption intensity and natural gas consumption intensity. (2) The impact of pollution charges on the end-of-pipe technology innovation of enterprises is linear (the square term of pollution charge is not significant). The influence of pollution charges on industrial wastewater discharge intensity, total energy intensity, and power consumption intensity is nonlinear, and there is a $U$ shaped relationship between them (the square coefficient of pollution charge is positive and the coefficient of pollution charge is negative). At the same time, the impact of pollution charges on the removal intensity of smoke and dust is not significant. (3) Although there is a $U$-shaped relationship between pollution charges and industrial wastewater discharge intensity, total energy intensity, and power consumption intensity, there are differences in the inflection points of the impact of pollution charges on the three types of green technology innovation. The curve inflection point of industrial wastewater discharge intensity is greater than that of the total energy consumption intensity or of the power consumption intensity.

From Tables 3 and 4, it can be seen that (1) pollution charges have pushed up the intensity of $\mathrm{SO}_{2}$ emissions to a certain extent (the regression coefficient between pollution charges and $\mathrm{SO}_{2}$ emission intensity is 0.357 ), while they have also improved the intensity of $\mathrm{SO}_{2}$ removal (the regression coefficient between pollution charges and $\mathrm{SO}_{2}$ removal intensity is 0.099 ), with a greater impact on the former than the latter; (2) pollution charges have pushed up the emission intensity of solid waste to a certain extent (the regression coefficient between industrial pollution charges and solid waste emission intensity is 0.162 ), and at the same time, they have also improved the removal intensity of solid waste (the regression coefficient between pollution charges and solid waste removal intensity is 0.066), with a greater impact on the former than the latter; (3) in a certain range, pollution charge reduces the intensity of industrial wastewater discharge (the regression coefficient between pollution charge sand industrial wastewater discharge intensity is -0.481), and at the same time, pollution charges also reduce the intensity of industrial wastewater removal (the regression coefficient between pollution charge and industrial wastewater removal intensity is -0.304); (4) electricity and fuel oil are the main energy sources produced by enterprises at the given time point, and they are also the main factors that determine the energy intensity, while coal and natural gas are not the main energy sources produced by enterprises at present. The above results show that pollution charges can promote the end-treatment of $\mathrm{SO}_{2}$, but inhibit the process improvement of $\mathrm{SO}_{2}$ emission reduction. However, the mechanism of pollution charges on industrial wastewater is the complete opposite; that is, pollution charges can inhibit the end-treatment of industrial wastewater, but promote the process improvement of industrial wastewater removal.

\subsection{Empirical Test Results in 2007}

Pollution charges at a given time have a certain influence on some types of green technology innovation by enterprises. (1) From Table 6, it can be seen that the pollution charges are only significantly correlated with the $\mathrm{SO}_{2}$ emission intensity and industrial wastewater emission intensity at the confidence levels of $1 \%$ and $10 \%$, respectively, and the regression coefficients are -3.583 and -3.447 , respectively. The regression coefficient of pollution charges, smoke and dust emission intensity, and water use intensity of enterprises is not significant. (2) We can see from Table 7 that there is a significant negative correlation between pollution charges and industrial wastewater removal intensity at the confidence level of $5 \%$, and the regression coefficient is -3.608 ; in addition, the regression coefficients of pollution charges with $\mathrm{SO}_{2}$ removal intensity and smoke dust removal intensity are not 
significant. (3) The econometric results given in Table 8 show that pollution charges are positively correlated with total energy consumption intensity, coal consumption intensity, and natural gas consumption intensity at the $1 \%$ confidence level, with regression coefficients of $0.416,0.340$, and 0.953 , respectively. There is a significant negative correlation between pollution charges and fuel oil consumption intensity at the confidence level of $1 \%$, and the regression coefficient is -0.853 .

Table 6. Regression results of the impact of pollution charges on enterprises' process improvement and technological innovation in 2007.

\begin{tabular}{|c|c|c|c|c|}
\hline & $\begin{array}{l}\mathrm{SO}_{2} \text { Emission } \\
\text { Intensity M14 }\end{array}$ & $\begin{array}{c}\text { Smoke and } \\
\text { Dust Emission } \\
\text { Intensity M15 }\end{array}$ & $\begin{array}{c}\text { Wastewater } \\
\text { Discharge } \\
\text { Intensity M16 }\end{array}$ & $\begin{array}{l}\text { Industrial Water } \\
\text { Intensity M17 }\end{array}$ \\
\hline \multirow[t]{2}{*}{$\begin{array}{l}\text { Pollution } \\
\text { charges }\end{array}$} & $-3.538^{* * *}$ & 1.174 & $-3.447^{*}$ & -0.880 \\
\hline & $(-2.60)$ & $(0.66)$ & $(-2.44)$ & $(-0.65)$ \\
\hline \multirow{2}{*}{$\begin{array}{l}\text { Square of } \\
\text { pollution charge }\end{array}$} & $2.145^{* * *}$ & -0.088 & $1.851^{* *}$ & 0.610 \\
\hline & (3.14) & $(-0.10)$ & $(2.61)$ & (1.09) \\
\hline \multirow{2}{*}{$\begin{array}{l}\text { Regional } \\
\text { industrial } \\
\text { structure }\end{array}$} & $-7.649 * * *$ & -1.614 & $-6.632 * * *$ & $-4.797^{* * *}$ \\
\hline & $(-7.67)$ & $(-1.26)$ & $(-6.27)$ & $(-4.88)$ \\
\hline VAT payable & $\begin{array}{l}0.274^{* * *} \\
(8.41)\end{array}$ & $\begin{array}{c}0.347^{* * *} \\
(8.09)\end{array}$ & $\begin{array}{c}0.130^{* * *} \\
(4.08)\end{array}$ & $\begin{array}{l}0.157^{* * *} \\
(5.22)\end{array}$ \\
\hline \multirow{2}{*}{$\begin{array}{l}\text { Gross industrial } \\
\text { output value }\end{array}$} & $-1.088^{* * *}$ & $-1.297^{* * *}$ & $-1.172^{* * *}$ & $-1.251^{* * *}$ \\
\hline & $(-6.34)$ & $(-5.75)$ & $(-6.61)$ & $(-7.62)$ \\
\hline Asset structure & $\begin{array}{c}1.536^{* * *} \\
(9.41)\end{array}$ & $\begin{array}{c}2.525^{* * *} \\
(11.94)\end{array}$ & $\begin{array}{c}0.849^{* * *} \\
(5.00)\end{array}$ & $\begin{array}{c}1.156^{* * * *} \\
(7.41)\end{array}$ \\
\hline \multirow{2}{*}{$\begin{array}{l}\text { Enterprise } \\
\text { employment }\end{array}$} & $-1.686^{* *}$ & $-3.500^{* * *}$ & $-2.235^{* *}$ & $-2.232^{* * *}$ \\
\hline & $(-2.46)$ & $(-3.93)$ & $(-3.17)$ & $(-3.32)$ \\
\hline Total asset & $\begin{array}{c}-0.0270 \\
(-1.03)\end{array}$ & $\begin{array}{l}-0.0124 \\
(-0.36)\end{array}$ & $\begin{array}{c}0.0822 \text { ** } \\
(3.12)\end{array}$ & $\begin{array}{c}0.119^{* * *} \\
(4.84)\end{array}$ \\
\hline Total profit & $\begin{array}{l}-0.0267 \\
(-1.63)\end{array}$ & $\begin{array}{c}-0.00916 \\
(-0.43)\end{array}$ & $\begin{array}{c}-0.0406 * \\
(-2.39)\end{array}$ & $\begin{array}{c}-0.0349 \text { ** } \\
(-2.21)\end{array}$ \\
\hline \multirow[t]{2}{*}{$\begin{array}{l}\text { Enterprise } \\
\text { intermediate } \\
\text { investment }\end{array}$} & 0.167 & 0.234 & $0.361 *$ & $0.532^{* * *}$ \\
\hline & (1.02) & (1.09) & $(2.15)$ & $(3.42)$ \\
\hline Enterprise age & $\begin{array}{c}-0.00120 \\
(-0.13)\end{array}$ & $\begin{array}{c}-0.0102 \\
(-0.82)\end{array}$ & $\begin{array}{c}-0.0175 \\
(-1.80)\end{array}$ & $\begin{array}{c}-0.0102 \\
(-1.12)\end{array}$ \\
\hline \multirow{2}{*}{$\begin{array}{l}\text { Annual normal } \\
\text { production time }\end{array}$} & $0.675^{* * *}$ & $0.786^{* * *}$ & $0.541^{* * *}$ & $0.583^{* * *}$ \\
\hline & (13.08) & (11.81) & (10.24) & (12.16) \\
\hline \multirow{2}{*}{$\begin{array}{l}\text { Employment } \\
\text { structure }\end{array}$} & $-1.486^{* * *}$ & $-2.114^{* * *}$ & $-1.016^{* * *}$ & $-1.127^{* * *}$ \\
\hline & $(-8.50)$ & $(-9.32)$ & $(-5.89)$ & $(-6.96)$ \\
\hline \multirow[t]{2}{*}{$\begin{array}{l}\text { Technology- } \\
\text { intensive } \\
\text { enterprise }\end{array}$} & $-0.128 *$ & -0.0694 & $-1.317^{* * *}$ & $-1.031^{* * *}$ \\
\hline & $(-1.72)$ & $(-0.71)$ & $(-17.91)$ & $(-14.88)$ \\
\hline \multirow{2}{*}{$\begin{array}{l}\text { Enterprise in the } \\
\text { eastern } \\
\text { provinces }\end{array}$} & $0.731^{* * *}$ & -0.0935 & $0.830^{* * *}$ & $0.484^{* * *}$ \\
\hline & $(6.97)$ & $(-0.69)$ & $(7.38)$ & $(4.64)$ \\
\hline$N$ & 3115 & 3119 & 3680 & 3963 \\
\hline$R^{2}$ & 0.265 & 0.250 & 0.225 & 0.189 \\
\hline
\end{tabular}

t-Statistics in parentheses; ${ }^{*} p<0.1,^{* *} p<0.05,{ }^{* * *} p<0.01$. 
Table 7. Regression results of the impact of pollution charges on enterprises' end-treatment technological innovation in 2007.

\begin{tabular}{|c|c|c|c|}
\hline & $\begin{array}{l}\mathrm{SO}_{2} \text { Removal } \\
\text { Intensity M18 }\end{array}$ & $\begin{array}{c}\text { Smoke and Dust } \\
\text { Removal Intensity } \\
\text { M19 }\end{array}$ & $\begin{array}{c}\text { Wastewater Removal } \\
\text { Intensity M20 }\end{array}$ \\
\hline Pollution charges & $\begin{array}{l}-1.555 \\
(-0.96)\end{array}$ & $\begin{array}{l}-1.257 \\
(-0.71)\end{array}$ & $\begin{array}{l}-3.608^{* *} \\
(-2.55)\end{array}$ \\
\hline \multirow{2}{*}{$\begin{array}{l}\text { Square of pollution } \\
\text { charge }\end{array}$} & 1.119 & 0.937 & $1.889^{* * *}$ \\
\hline & (1.37) & $(1.06)$ & $(2.65)$ \\
\hline $\begin{array}{l}\text { Regional industrial } \\
\text { structure }\end{array}$ & $-4.001 * *$ & $-2.967^{* *}$ & $-5.764^{* * *}$ \\
\hline & $(-2.55)$ & $(-2.28)$ & $(-5.25)$ \\
\hline VAT payable & $\begin{array}{c}0.208^{* * *} \\
(3.68)\end{array}$ & $\begin{array}{c}0.369 * * * \\
(7.96)\end{array}$ & $\begin{array}{c}0.133^{* * *} \\
(4.00)\end{array}$ \\
\hline \multirow{2}{*}{$\begin{array}{l}\text { Gross industrial } \\
\text { output value }\end{array}$} & $-0.948^{* * *}$ & $-1.303^{* * *}$ & $-1.113^{* * *}$ \\
\hline & $(-3.27)$ & $(-5.52)$ & $(-5.73)$ \\
\hline Asset structure & $\begin{array}{c}1.584 * * * \\
(5.76)\end{array}$ & $\begin{array}{l}2.211^{* * * *} \\
(9.91)\end{array}$ & $\begin{array}{l}1.015^{* * * *} \\
(5.63)\end{array}$ \\
\hline \multirow{2}{*}{$\begin{array}{l}\text { Enterprise } \\
\text { employment }\end{array}$} & -0.914 & $-3.406^{* * *}$ & $-2.581^{* * *}$ \\
\hline & $(-1.13)$ & $(-3.93)$ & $(-3.75)$ \\
\hline Total asset & $\begin{array}{l}-0.0106 \\
(-0.24)\end{array}$ & $\begin{array}{l}0.0538 \\
(1.47)\end{array}$ & $\begin{array}{c}0.0501 \text { * } \\
(1.84)\end{array}$ \\
\hline Total profit & $\begin{array}{l}0.0256 \\
(0.86)\end{array}$ & $\begin{array}{l}0.0226 \\
(1.04)\end{array}$ & $\begin{array}{c}-0.0517^{* * *} \\
(-2.90)\end{array}$ \\
\hline \multirow[t]{2}{*}{$\begin{array}{l}\text { Enterprise } \\
\text { intermediate } \\
\text { investment }\end{array}$} & 0.0468 & 0.217 & 0.277 \\
\hline & $(0.17)$ & $(0.96)$ & (1.51) \\
\hline Enterprise age & $\begin{array}{l}-0.0142 \\
(-0.93)\end{array}$ & $\begin{array}{c}-0.00545 \\
(-0.42)\end{array}$ & $\begin{array}{l}-0.0143 \\
(-1.37)\end{array}$ \\
\hline \multirow{2}{*}{$\begin{array}{l}\text { Annual normal } \\
\text { production time }\end{array}$} & $0.540^{* * *}$ & $0.714^{* * *}$ & $0.500^{* * *}$ \\
\hline & $(6.35)$ & $(10.07)$ & $(9.07)$ \\
\hline \multirow[t]{2}{*}{$\begin{array}{l}\text { Employment } \\
\text { structure }\end{array}$} & $-1.245^{* * *}$ & $-1.620^{* * *}$ & $-1.075^{* * *}$ \\
\hline & $(-4.25)$ & $(-6.79)$ & $(-5.90)$ \\
\hline \multirow[t]{2}{*}{$\begin{array}{l}\text { Technology-intensive } \\
\text { enterprise }\end{array}$} & $-0.256^{* *}$ & 0.146 & $-1.261^{* * *}$ \\
\hline & $(2.10)$ & $(1.41)$ & $(-16.33)$ \\
\hline \multirow{2}{*}{$\begin{array}{l}\text { Enterprise in the } \\
\text { eastern provinces }\end{array}$} & $0.308 *$ & 0.115 & $0.611^{* * *}$ \\
\hline & $(1.73)$ & $(0.83)$ & $(4.96)$ \\
\hline$N$ & 1452 & 2460 & 2971 \\
\hline$R^{2}$ & 0.196 & 0.226 & 0.259 \\
\hline
\end{tabular}

The influence of pollution charges on different types of green technology innovation at a given time is inconsistent. (1) From Tables 6-8, we can see that on the whole, pollution charges can promote some process improvement and end-of-pipe technology innovation, and can have a negative impact on most energy-saving green technology innovation. (2) The impact of pollution charges on energy-saving green technology innovation is linear (the square term of pollution charges is not significant). The influence of pollution charges on $\mathrm{SO}_{2}$ emission intensity, industrial wastewater emission intensity, and industrial wastewater removal intensity is nonlinear, and there is a $U$-shaped relationship between them (the square coefficient of pollution charges is positive and the coefficient of pollution charges is negative). (3) Although there is a $U$-shaped relationship between pollution charges and 
$\mathrm{SO}_{2}$ emission intensity, industrial wastewater emission intensity, and industrial wastewater removal intensity, the inflexion points of pollution charges on the three kinds of green technological innovations are different. $\mathrm{SO}_{2}$ emission intensity is less than industrial wastewater emission intensity and industrial wastewater removal intensity.

Table 8. Regression results of the impact of pollution charges on enterprises' energy-saving technological innovation in 2007.

\begin{tabular}{|c|c|c|c|c|}
\hline & $\begin{array}{c}\text { Energy } \\
\text { Consumption } \\
\text { Intensity M21 }\end{array}$ & $\begin{array}{c}\text { Enterprise Coal } \\
\text { Intensity M22 }\end{array}$ & $\begin{array}{c}\text { Enterprise Fuel } \\
\text { Oil Intensity } \\
\text { M23 }\end{array}$ & $\begin{array}{c}\text { Enterprise } \\
\text { Natural Gas } \\
\text { Intensity M24 }\end{array}$ \\
\hline $\begin{array}{l}\text { Pollution } \\
\text { charges }\end{array}$ & $\begin{array}{c}0.416^{* * *} \\
(4.85)\end{array}$ & $\begin{array}{c}0.340 * * * \\
(14.32)\end{array}$ & $\begin{array}{c}-0.853^{* * *} \\
(-8.05)\end{array}$ & $\begin{array}{c}0.953^{* * *} \\
(6.16)\end{array}$ \\
\hline $\begin{array}{l}\text { Square of } \\
\text { pollution charge }\end{array}$ & $\begin{array}{l}-0.008 \\
(-1.01)\end{array}$ & $\begin{array}{l}-0.001 \\
(-1.09)\end{array}$ & $\begin{array}{l}2.361 \\
(0.02)\end{array}$ & $\begin{array}{l}-0.0652 \\
(-0.09)\end{array}$ \\
\hline $\begin{array}{l}\text { Regional } \\
\text { industrial } \\
\text { structure }\end{array}$ & $\begin{array}{c}-2.326^{* * *} \\
(-2.71)\end{array}$ & $\begin{array}{c}-1.675^{* * *} \\
(-6.99)\end{array}$ & $\begin{array}{l}-0.760 \\
(-0.83)\end{array}$ & $\begin{array}{l}-0.934 \\
(-0.81)\end{array}$ \\
\hline VAT payable & $\begin{array}{c}0.248^{* * * *} \\
(8.71)\end{array}$ & $\begin{array}{c}0.269^{* * *} \\
(26.10)\end{array}$ & $\begin{array}{l}0.124^{* * * *} \\
(3.91)\end{array}$ & $\begin{array}{c}0.300^{* * *} \\
(5.42)\end{array}$ \\
\hline $\begin{array}{l}\text { Gross industrial } \\
\text { output value }\end{array}$ & $\begin{array}{c}-0.926^{* * *} \\
(-6.45)\end{array}$ & $\begin{array}{l}-1.106^{* * *} \\
(-22.36)\end{array}$ & $\begin{array}{l}-0.0530 \\
(-0.32)\end{array}$ & $\begin{array}{c}-1.124^{* * *} \\
(-5.00)\end{array}$ \\
\hline Asset structure & $\begin{array}{c}1.817^{* * * *} \\
(12.99)\end{array}$ & $\begin{array}{c}1.362^{* * *} \\
(27.00)\end{array}$ & $\begin{array}{c}0.736^{* * * *} \\
(3.93)\end{array}$ & $\begin{array}{c}1.399^{* * * *} \\
(4.22)\end{array}$ \\
\hline $\begin{array}{l}\text { Enterprise } \\
\text { employment }\end{array}$ & $\begin{array}{c}-1.655^{* * *} \\
(-5.51)\end{array}$ & $\begin{array}{l}-0.504^{* *} \\
(-2.97)\end{array}$ & $\begin{array}{l}0.102 \\
(0.31)\end{array}$ & $\begin{array}{c}-1.903^{* * *} \\
(-5.17)\end{array}$ \\
\hline Total asset & $\begin{array}{c}0.00301 \\
(0.13)\end{array}$ & $\begin{array}{c}0.0867^{* * *} \\
(10.09)\end{array}$ & $\begin{array}{l}-0.0119 \\
(-0.42)\end{array}$ & $\begin{array}{l}-0.0367 \\
(-0.71)\end{array}$ \\
\hline Total profit & $\begin{array}{l}-0.0224 \\
(-1.59)\end{array}$ & $\begin{array}{c}-0.0246^{* * *} \\
(-4.94)\end{array}$ & $\begin{array}{c}-0.0629 * * * \\
(-3.32)\end{array}$ & $\begin{array}{l}-0.0366 \\
(-1.05)\end{array}$ \\
\hline $\begin{array}{l}\text { Enterprise } \\
\text { intermediate } \\
\text { investment }\end{array}$ & 0.0425 & $0.0909 *$ & $-0.484^{* * *}$ & 0.242 \\
\hline Enterprise age & $\begin{array}{l}(0.31) \\
0.0119 \\
(1.42)\end{array}$ & $\begin{array}{c}(1.92) \\
-0.000373 \\
(-0.13)\end{array}$ & $\begin{array}{c}(-3.07) \\
-0.00287 \\
(-0.27)\end{array}$ & $\begin{array}{c}(1.17) \\
-0.0820 * * * \\
(-4.45)\end{array}$ \\
\hline $\begin{array}{l}\text { Annual normal } \\
\text { production time }\end{array}$ & $\begin{array}{c}0.736^{* * *} \\
(16.33)\end{array}$ & $\begin{array}{c}0.970 * * * \\
(60.98)\end{array}$ & $\begin{array}{l}0.660 * * * \\
(11.68)\end{array}$ & $\begin{array}{c}0.837^{* * * *} \\
(9.06)\end{array}$ \\
\hline $\begin{array}{l}\text { Employment } \\
\text { structure }\end{array}$ & $\begin{array}{l}-1.615^{* * *} \\
(-10.91)\end{array}$ & $\begin{array}{l}-1.263^{* * *} \\
(-23.81)\end{array}$ & $\begin{array}{c}-0.441^{* *} \\
(-2.39)\end{array}$ & $\begin{array}{l}-0.443 \\
(-1.28)\end{array}$ \\
\hline $\begin{array}{l}\text { Technology- } \\
\text { intensive } \\
\text { enterprise }\end{array}$ & $\begin{array}{l}-0.0963 \\
(-1.50)\end{array}$ & $\begin{array}{c}-0.151^{* * *} \\
(-6.57)\end{array}$ & $\begin{array}{l}0.0863 \\
(1.03)\end{array}$ & $\begin{array}{l}0.210 \\
(1.25)\end{array}$ \\
\hline $\begin{array}{l}\text { Enterprise in the } \\
\text { eastern } \\
\text { provinces }\end{array}$ & $\begin{array}{c}0.348^{* * *} \\
(3.84)\end{array}$ & $\begin{array}{l}-0.0120 \\
(-0.44)\end{array}$ & $\begin{array}{c}0.861^{* * *} \\
(6.88)\end{array}$ & $\begin{array}{l}0.283 * \\
(1.82)\end{array}$ \\
\hline $\begin{array}{l}N \\
R^{2}\end{array}$ & $\begin{array}{l}3385 \\
0.296\end{array}$ & $\begin{array}{c}22,011 \\
0.369\end{array}$ & $\begin{array}{l}3192 \\
0.207\end{array}$ & $\begin{array}{l}1343 \\
0.238\end{array}$ \\
\hline
\end{tabular}


There are some common laws in the influence of pollution charges on different types of green technology innovation at a given time. From Tables 6 and 7, we can see that (1) within a certain range, pollution charges reduce industrial wastewater discharge intensity to a certain extent (the regression coefficient between pollution charges and industrial wastewater discharge intensity is -3.447 ), while pollution charges also reduce industrial wastewater removal intensity (the regression coefficient between pollution charges and industrial wastewater removal intensity is -3.608). (2) Pollution charges have no significant impact on the removal intensity and emission intensity of smoke and dust; that is, as far as smoke and dust are concerned, the collection of pollution charges does not significantly promote the technological innovation of emissions reduction at the source, nor does it significantly promote the technological innovation of emissions reduction at the end. (3) Coal and natural gas are the main energy sources produced by enterprises at the given time point, and they are also the main factors that determine the energy intensity, while fuel oil is not the main energy source produced by enterprises at present-that is, pollution charges can reduce the fuel oil consumption intensity, but can improve the total energy consumption intensity, so it is very likely that the intensity of coal and natural gas use is increased. The above results show that the pollution charges can inhibit the end-treatment of industrial wastewater, but can promote improvement of the process of industrial wastewater emission reduction.

\subsection{Discussion}

(1) The effectiveness of pollution charges on the green technology innovation of enterprises has been significantly improved. From the above empirical results, it can be seen that in 2012, the pollution charges had no significant effect on the intensity of smoke and dust removal, but had significant effects on other end-of-pipe technology innovation, green process innovation, and energy-saving green technology innovation of enterprises; that is, the government has imposed pollution discharge fees on enterprises, causing a certain impact on various green technology innovations of enterprises. At the same time, observing the empirical results for 2007, we can see that pollution charges had no significant effect on the intensity of smoke and dust removal, but had significant effects on the intensity of water production, $\mathrm{SO}_{2}$ removal, and smoke and dust emissions; we can find similar rules for the degree of significance. Therefore, we can confirm that with the passage of time, the efficiency and validity of pollution charges in promoting green technology innovation by enterprises have been improved to a certain extent. At the same time, with the development of the economy and society, the eastern provinces and technology-intensive industries have an increasingly significant influence on the green technology innovation of process improvement;

(2) The regulation of pollution charges reduces the discharge intensity and removal intensity of industrial wastewater. From Tables $3-5$ and Table 7, we can see that the regression coefficients of pollution charges and industrial wastewater discharge intensity and removal intensity are all negative; that is, pollution charges reduce industrial wastewater discharge intensity to a certain extent (the regression coefficients of pollution charges and industrial wastewater discharge intensity for 2007 and 2012 are -0.481 and -3.447 , respectively), and pollution charges also reduce industrial wastewater removal intensity. The above results show that, for industrial wastewater, pollution charges can inhibit the end-of-pipe technology innovation, but can promote the process improvement of industrial wastewater emission reduction;

(3) There is a nonlinear dynamic relationship between pollution charges and green technology innovation. In the empirical results for 2007 and 2012, the influence of pollution charges on the emission intensity of smoke and dust was not significant, and because of statistical data, there was no record of solid waste discharge intensity in 2007, so this was not the object of follow-up analysis. From Tables 3-8, we can see that there is a $U$-shaped relationship between pollution charges and enterprises' green technology innovation. Specifically, pollution charges have a $U$-shaped influence on industrial wastewater 
discharge intensity and industrial wastewater removal intensity; that is, in terms of industrial wastewater removal intensity and discharge intensity, the current regulation has not reached the inflection point, and moderately improving the regulation of wastewater removal will further improve the technological innovation ability of enterprises in this respect. In 2007, the relationship between pollution charges and $\mathrm{SO}_{2}$ emission intensity was $U$-shaped; that is, the intensity of $\mathrm{SO}_{2}$ emissions could be reduced by increasing the intensity of pollution charges within a certain range. However, the regression results for 2012 show that there was a linear increase between pollution charges and $\mathrm{SO}_{2}$ emission intensity; that is, pollution charges pushed up the intensity of $\mathrm{SO}_{2}$ emissions, largely because the regulation intensity had exceeded the inflection point (pollution charges squeezed the investment in green technology innovation of enterprises), so it was necessary to appropriately adjust the regulation of $\mathrm{SO}_{2}$ emission intensity;

(4) The impacts of enterprise asset structure and depreciation on different types of green technology innovation are quite different. Although there are some differences in the selection of control variables between the empirical models in 2007 and 2012, we found that there was a significant positive correlation between asset structure and waste emission intensity (e.g., $\mathrm{SO}_{2}$ emission intensity, smoke and dust emission intensity, solid waste emission intensity, industrial wastewater emission intensity), energy intensity, and enterprise production water intensity; that is, the higher the proportion of fixed assets, the higher the cost of process improvement for enterprises, because process improvement involves modification and replacement of existing production processes and production equipment. Therefore, the excessive proportion of fixed assets will reduce the enthusiasm of enterprises for green technology innovation; instead, they will choose end-of-pipe technology innovation, and the empirical results have been well verified. The greater the accumulated depreciation, the greater the risk of equipment investment; therefore, enterprises with greater accumulated depreciation are less motivated to improve production technology and update production equipment to reduce emissions. On the other hand, enterprises with greater depreciation tend to choose end-of-pipe technological innovation to achieve the legitimacy of enterprise production and reduce the environmental cost of enterprise production. We can see that larger solid assets of enterprises and faster depreciation of assets will inhibit enterprises from adopting the green process innovation, and promote enterprises to adopt the end-of-pipe technological innovation.

\section{Conclusions}

This article obtained basic data by matching China's Industrial Enterprise Database, China's Industrial Enterprise Pollution Emission Database, and regional pollution charges. Based on these data, 24 empirical models were constructed to analyze the impacts of pollution charges on different types green technology innovation by enterprises in 2007 and 2012. The results are consistent with our preliminary assumption, indicating that the pollution charges will indeed have a certain impact on the green technology innovation of enterprises. Such impact is not invariable; it is reflected in the type of green technology innovation and the intensity of the charges, as well as the time. At the same time, it enables the moderation of the internal characteristics of enterprises, including solid assets, asset depreciation, location, and industry. From the above research, the following conclusions can be obtained:

(1) The effectiveness of pollution charges on all kinds of green technology innovation by enterprises has significantly improved with the passage of time. This is consistent with the findings of Sellitto et al. and Porter [50,52], and will not change due to the difference in research subjects and methods. At the same time, with economic and social development, the impact of eastern provinces and technology-intensive industries on green process innovation is becoming more and more significant;

(2) Pollution charges can inhibit the end-of-pipe technology innovation for end-treatment of industrial wastewater, but can promote the process improvement of industrial wastewater emission reduction. This shows that the role of pollution charges in indus- 
trial sewage treatment comes from process improvement. The "inhibition hypothesis" holds that pollution charges will increase costs to enterprises, reflected mainly in the increase in external pollution control costs and the increased innovation investment to adapt to the current policies $[42,56]$;

(3) There is a nonlinear relationship between pollution charges and enterprises' green technology innovation, and it is constantly adjusted with the change in time. There is a $U$-shaped relationship between pollution charges and enterprises' green technology innovation (i.e., $\mathrm{SO}_{2}$ emission intensity, industrial wastewater emission intensity, and industrial wastewater removal intensity). Sanchez-Vargas et al. [57] asserted that the relationship between environmental regulation and productivity is actually nonlinear. One explanation of the "uncertainty hypothesis" is that, in the short term, investment related to pollution control will crowd out green technology R\&D investment, but in the long term, the compensatory effect of innovation can generate additional profits to promote technological progress [58];

(4) The larger the enterprise's solid assets, the faster the asset depreciation will inhibit the enterprise from adopting a green process innovation strategy. On the contrary, it will encourage the enterprise to adopt an end-pipe technology innovation strategy, meaning that the internal characteristics of the enterprise have a moderate effect on the role of pollution charges.

Therefore, we should not reduce the intensity of environmental regulations because of the current downward trajectory of the economy. Some local governments are trying to stimulate economic recovery by reducing the intensity of environmental regulations or starting high-carbon projects, which will have a negative impact on China's green economic recovery. The Porter hypothesis highlights how appropriate environmental regulation will stimulate enterprises' green technology innovation, so as to offset the environmental cost of production and, finally, improve enterprises' market competitiveness. The conclusion of this article further confirms this theoretical hypothesis. Therefore, at present, we cannot reduce the intensity of environmental regulations; instead, we should focus on the design and implementation of regulatory measures to drive enterprises' green technology innovation.

Nevertheless, this article has some limitations, which need to be improved in future research. Firstly, the data used in this article were cross-sectional data, which cannot capture the influence of time changes. Secondly, the model used in this article is the ROLS; although enough factors are controlled, the endogeneity still may not be ignored. Finally, the measurement variables of green technology innovation need to be further unified and standardized.

Author Contributions: All authors contributed to the study conception and design. Material preparation, data collection, and analysis were performed by M.W., Y.L., J.L. and Z.W. The first draft of the manuscript was written by M.W., and all authors commented on previous versions of the manuscript. All authors have read and agreed to the published version of the manuscript.

Funding: This work was supported by the China Postdoctoral Science Foundation (2020M670470).

Institutional Review Board Statement: Not applicable.

Informed Consent Statement: Informed consent was obtained from all subjects involved in the study.

Data Availability Statement: The datasets used and/or analyzed during the current study are available from the corresponding author on reasonable request.

Conflicts of Interest: The authors have no relevant financial or non-financial interests to disclose.

\section{References}

1. Geng, G.; Zheng, Y.; Zhang, Q.; Xue, T.; Zhao, H.; Tong, D.; Zheng, B.; Li, M.; Liu, F.; Hong, C.; et al. Drivers of PM2.5 air pollution deaths in China 2002-2017. Nat. Geosci. 2021, 14, 645-650. [CrossRef]

2. Zhang, Q.; He, K.; Hong, H. Cleaning China's air. Nature 2012, 484, 161-162. [CrossRef] [PubMed]

3. Wang, M.; Li, Y.; Li, M.; Wan, L.; Miao, L.; Wang, X. A comparative study on recycling amount and rate of used products under different regulatory scenarios. J. Clean. Prod. 2019, 235, 1153-1169. [CrossRef] 
4. Zhan, Y.; Tan, K.H.; Ji, G.; Chung, L.; Chiu, A.S.F. Green and lean sustainable development path in China: Guanxi, practices and performance. Resour. Conserv. Recycl. 2018, 128, 240-249. [CrossRef]

5. Zhang, J.; Zhang, N.; Bai, S. Assessing the carbon emission changing for sustainability and high-quality economic development. Environ. Technol. Innov. 2021, 22, 101464. [CrossRef]

6. Lin, B.; Zhu, J. The role of renewable energy technological innovation on climate change: Empirical evidence from China. Sci. Total Environ. 2019, 659, 1505-1512. [CrossRef]

7. Wang, M.Y.; Li, Y.; Cheng, Z.; Zhong, C.; Ma, W. Evolution and equilibrium of a green technological innovation system: Simulation of a tripartite game model. J. Clean. Prod. 2021, 278, 123944. [CrossRef]

8. Wang, Q.; Qu, J.; Wang, B.; Wang, P.; Yang, T. Green technology innovation development in China in 1990-2015. Sci. Total Environ. 2019, 696, 134008. [CrossRef]

9. Wang, M.; Li, Y.; Li, J.; Wang, Z. Green process innovation, green product innovation and its economic performance improvement paths: A survey and structural model. J. Environ. Manag. 2021, 297, 113282. [CrossRef]

10. Wang, Z.; Wang, Q.; Chen, B.; Wang, Y. Evolutionary game analysis on behavioral strategies of multiple stakeholders in E-waste recycling industry. Resour. Conserv. Recycl. 2020, 155, 104618. [CrossRef]

11. Da Silva Rocha, A.B.; Salomão, G.M. Environmental policy regulation and corporate compliance in evolutionary game models with well-mixed and structured populations. Eur. J. Oper. Res. 2019, 279, 486-501. [CrossRef]

12. Dewick, P.; Maytorena-Sanchez, E.; Winch, G. Regulation and regenerative eco-innovation: The case of extracted materials in the UK. Ecol. Econ. 2019, 160, 38-51. [CrossRef]

13. Harford, J.D. Firm behavior under imperfectly enforceable pollution standards and taxes. J. Environ. Econ. Manag. 1978, 5, 26-43. [CrossRef]

14. Horbach, J. Determinants of environmental innovation-New evidence from German panel data sources. Res. Policy 2008, 37, 163-173. [CrossRef]

15. Fu, S.; Ma, Z.; Ni, B.; Peng, J.; Zhang, L.; Fu, Q. Research on the spatial differences of pollution-intensive industry transfer under the environmental regulation in China. Ecol. Indic. 2021, 129, 107921. [CrossRef]

16. Yang, Y.; Zhao, L.; Wang, C.; Xue, J. Towards more effective air pollution governance strategies in China: A systematic review of the literature. J. Clean. Prod. 2021, 297, 126724. [CrossRef]

17. Streimikiene, D. Review of internalization of externalities and dynamics of atmospheric emissions in energy sector of Baltic States. Renew. Sustain. Energy Rev. 2017, 70, 1131-1141. [CrossRef]

18. Nguyen, T.L.T.; Laratte, B.; Guillaume, B.; Hua, A. Quantifying environmental externalities with a view to internalizing them in the price of products, using different monetization models. Resour. Conserv. Recycl. 2016, 109, 13-23. [CrossRef]

19. Clarkson, P.M.; Li, Y.; Richardson, G.D. The Market Valuation of Environmental Capital Expenditures by Pulp and Paper Companies. Account. Rev. 2004, 79, 329-353. [CrossRef]

20. Brunel, C. Green innovation and green Imports:Links between environmental policies, innovation, and production. J. Environ. Manag. 2019, 248, 109290. [CrossRef]

21. Hazarika, N.; Zhang, X. Factors that drive and sustain eco-innovation in the construction industry: The case of Hong Kong. $J$. Clean. Prod. 2019, 238, 117816. [CrossRef]

22. Yusoff, Y.M.; Omar, M.K.; Kamarul Zaman, M.D.; Samad, S. Do all elements of green intellectual capital contribute toward business sustainability? Evidence from the Malaysian context using the Partial Least Squares method. J. Clean. Prod. 2019, 234, 626-637. [CrossRef]

23. Norberg-Bohm, V. Stimulating 'green' technological innovation: An analysis of alternative policy mechanisms. Policy Sci. 1999, 32, 13-38. [CrossRef]

24. Xu, L.; Zhang, Q.; Shi, X. Stakeholders strategies in poverty alleviation and clean energy access: A case study of China's PV poverty alleviation program. Energy Policy 2019, 135, 111011. [CrossRef]

25. Romer, P.M. Endogenous Technological Change. J. Polit. Econ. 1990, 98, S71-S102. [CrossRef]

26. Abbas, J.; Sağsan, M. Impact of knowledge management practices on green innovation and corporate sustainable development: A structural analysis. J. Clean. Prod. 2019, 229, 611-620. [CrossRef]

27. Luo, Q.; Miao, C.; Sun, L.; Meng, X.; Duan, M. Efficiency evaluation of green technology innovation of China's strategic emerging industries: An empirical analysis based on Malmquist-data envelopment analysis index. J. Clean. Prod. 2019, $238,117782$. [CrossRef]

28. Sun, H.; Edziah, B.K.; Sun, C.; Kporsu, A.K. Institutional quality, green innovation and energy efficiency. Energy Policy 2019, 135, 111002. [CrossRef]

29. Tariq, A.; Badir, Y.F.; Tariq, W.; Bhutta, U.S. Drivers and consequences of green product and process innovation: A systematic review, conceptual framework, and future outlook. Technol. Soc. 2017, 51, 8-23. [CrossRef]

30. Roper, S.; Tapinos, E. Taking risks in the face of uncertainty: An exploratory analysis of green innovation. Technol. Forecast. Soc. Chang. 2016, 112, 357-363. [CrossRef]

31. Wu, D.; Kefan, X.; Hua, L.; Shi, Z.; Olson, D.L. Modeling technological innovation risks of an entrepreneurial team using system dynamics: An agent-based perspective. Technol. Forecast. Soc. Chang. 2010, 77, 857-869. [CrossRef]

32. Brav, A.; Jiang, W.; Ma, S.; Tian, X. How does hedge fund activism reshape corporate innovation? J. Financ. Econ. 2018, 130, 237-264. [CrossRef] 
33. Fernández Fernández, Y.; Fernández López, M.A.; Olmedillas Blanco, B. Innovation for sustainability: The impact of R\&D spending on CO2 emissions. J. Clean. Prod. 2018, 172, 3459-3467. [CrossRef]

34. Zhao, S.; Jiang, Y.; Wang, S. Innovation stages, knowledge spillover, and green economy development: Moderating role of absorptive capacity and environmental regulation. Environ. Sci. Pollut. Res. 2019, 26, 25312-25325. [CrossRef]

35. Marino, M.; Parrotta, P.; Valletta, G. Electricity (de)regulation and innovation. Res. Policy 2019, 48, 748-758. [CrossRef]

36. Sgroi, F. Territorial development models: A new strategic vision to analyze the relationship between the environment, public goods and geographical indications. Sci. Total Environ. 2021, 787, 147585. [CrossRef]

37. Cohen, F.; Glachant, M.; Söderberg, M. The impact of energy prices on product innovation: Evidence from the UK refrigerator market. Energy Econ. 2017, 68, 81-88. [CrossRef]

38. Wang, M.; Cheng, Z.; Li, Y.; Li, J.; Guan, K. Impact of market regulation on economic and environmental performance: A game model of endogenous green technological innovation. J. Clean. Prod. 2020, 277, 123969. [CrossRef]

39. Beria, P. The role of transport supply in the acceptability of pollution charge extension. The case f Milan: A rejoinder. Transp. Res. Part A Policy Pract. 2020, 133, 381-382. [CrossRef]

40. Petroni, G.; Bigliardi, B.; Galati, F. Rethinking the Porter Hypothesis: The Underappreciated Importance of Value Appropriation and Pollution Intensity. Rev. Policy Res. 2018, 36, 121-140. [CrossRef]

41. Saunila, M.; Rantala, T.; Ukko, J.; Havukainen, J. Why invest in green technologies? Sustainability engagement among small businesses. Technol. Anal. Strat. Manag. 2019, 31, 653-666. [CrossRef]

42. Wagner, M. On the relationship between environmental management, environmental innovation and patenting: Evidence from German manufacturing firms. Res. Policy 2007, 36, 1587-1602. [CrossRef]

43. Yang, G.; Zha, D.; Wang, X.; Chen, Q. Exploring the nonlinear association between environmental regulation and carbon intensity in China: The mediating effect of green technology. Ecol. Indic. 2020, 114, 106309. [CrossRef]

44. Berrone, P.; Fosfuri, A.; Gelabert, L.; Gomez-Mejia, L. Necessity as the mother of "green" inventions: Institutional pressures and environmental innovations. Strateg. Manag. J. 2013, 34, 891-909. [CrossRef]

45. Qi, G.; Zeng, S.; Tam, C.; Yin, H.; Zou, H. Stakeholders' influences on corporate green innovation strategy: A case study of manufacturing firms in China. Corp. Soc. Responsib. Environ. Manag. 2013, 20, 1-14. [CrossRef]

46. Henriques, I.; Sadorsky, P. The Determinants of an Environmentally Responsive Firm: An Empirical Approach. J. Environ. Econ. Manag. 1996, 30, 381-395. [CrossRef]

47. Pansera, M.; Owen, R. Framing inclusive innovation within the discourse of development: Insights from case studies in India. Res. Policy 2018, 47, 23-34. [CrossRef]

48. Grossman, G. Growth, Trade, and Inequality. Econometrica 2018, 86, 37-83. [CrossRef]

49. Ambec, S.; Barla, P. A theoretical foundation of the Porter hypothesis. Econ. Lett. 2002, 75, 355-360. [CrossRef]

50. Sellitto, M.A.; Camfield, C.G.; Buzuku, S. Green innovation and competitive advantages in a furniture industrial cluster: A survey and structural model. Sustain. Prod. Consum. 2020, 23, 94-104. [CrossRef]

51. Tu, Y.; Wu, W. How does green innovation improve enterprises' competitive advantage? The role of organizational learning. Sustain. Prod. Consum. 2021, 26, 504-516. [CrossRef]

52. Porter, M.E. America's green strategy. Sci. Am. 1991, 264, 168. [CrossRef]

53. Brandt, L.; Van Biesebroeck, J.; Zhang, Y. Creative accounting or creative destruction? Firm-level productivity growth in Chinese manufacturing. J. Dev. Econ. 2012, 97, 339-351. [CrossRef]

54. Zhang, H.; Zhang, J.; He, Y. Are There Inter-enterprises Total Factor Productivity Spillover? An Investigation Based on China's Industrial Enterprises Data. Nankai Econ. Stud. 2018, 202, 102-119. [CrossRef]

55. Lu, J.; Tao, Z. Trends and determinants of China's industrial agglomeration. J. Urban Econ. 2009, 65, 167-180. [CrossRef]

56. Chintrakarn, P. Environmental regulation and U.S. states' technical inefficiency. Econ. Lett. 2008, 100, 363-365. [CrossRef]

57. Sanchez-Vargas, A.; Mansilla-Sanchez, R.; Aguilar-Ibarra, A. An Empirical Analysis of the Nonlinear Relationship Between Environmental Regulation and Manufacturing Productivity. J. Appl. Econ. 2013, 16, 357-372. [CrossRef]

58. Lanoie, P.; Patry, M.; Lajeunesse, R. Environmental regulation and productivity: Testing the porter hypothesis. J. Product. Anal. 2008, 30, 121-128. [CrossRef] 